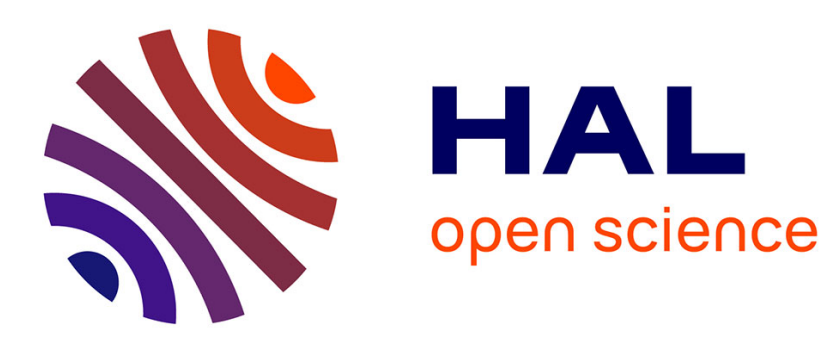

\title{
Macroscopically consistent non-local modelling of heterogeneous media
}

François Bignonnet, Karam Sab, Luc Dormieux, Sébastien Brisard, Antoine Bisson

\section{- To cite this version:}

François Bignonnet, Karam Sab, Luc Dormieux, Sébastien Brisard, Antoine Bisson. Macroscopically consistent non-local modelling of heterogeneous media. Computer Methods in Applied Mechanics and Engineering, 2014, 278, pp.218 - 238. 10.1016/j.cma.2014.05.014 . hal-01076820

\section{HAL Id: hal-01076820 \\ https://hal-enpc.archives-ouvertes.fr/hal-01076820}

Submitted on 23 Oct 2014

HAL is a multi-disciplinary open access archive for the deposit and dissemination of scientific research documents, whether they are published or not. The documents may come from teaching and research institutions in France or abroad, or from public or private research centers.
L'archive ouverte pluridisciplinaire HAL, est destinée au dépôt et à la diffusion de documents scientifiques de niveau recherche, publiés ou non, émanant des établissements d'enseignement et de recherche français ou étrangers, des laboratoires publics ou privés. 
NOTICE: this is the author's version of a work that was accepted for publication in Computer Methods in Applied Mechanics and Engineering. Changes resulting from the publishing process, such as peer review, editing, corrections, structural formatting, and other quality control mechanisms may not be reflected in this document. Changes may have been made to this work since it was submitted for publication. A definitive version was subsequently published in Computer Methods in Applied Mechanics and Engineering Vol. 278 p.218-238 (2014) http://dx.doi.org/10.1016/j.cma.2014.05.014

\title{
Macroscopically consistent non-local modelling of heterogeneous media
}

\author{
François Bignonnet, Karam Sab*, Luc Dormieux, Sébastien Brisard, Antoine Bisson \\ Université Paris-Est, Laboratoire Navier (UMR 8205), CNRS, ENPC, IFSTTAR, F-77455 Marne-la-Vallée, France
}

\begin{abstract}
Within the framework of the homogenization of heterogeneous media, a non local model is proposed. A field of non-local filtered stiffness tensor is introduced by filtering the solution to the homogenization problem. The filtered stiffness tensor, depending on the filter to heterogeneity size ratio, provides a continuous transition from the actual micro-scale heterogeneous stiffness field to the macro-scale homogenized stiffness tensor. For any intermediate filter size, the homogenization of the filtered stiffness yields exactly the homogenized stiffness, therefore it is called macroscopically consistent. The non-local stiffness tensor is intrinsically non symmetric, but its spatial fluctuations are smoothed, allowing for a less refined discretization in numerical methods. As a by-product, a two step heterogeneous multiscale method is proposed to reduce memory and computational time requirements of existing direct schemes while controlling the accuracy of the result. The first step is the estimation of the filtered stiffness at sampling points by means of an oversampling strategy to reduce boundary effects. The second step is the numerical homogenization of the obtained sampled filtered stiffness.
\end{abstract}

Keywords: Homogenization, Non-local modelling, Filtering, Heterogeneous Multiscale Method, Oversampling

\section{Introduction}

The numerical homogenization of the mechanical elastic properties of a heterogeneous medium is considered, or Heterogeneous Multiscale Methods (HMM) as referred to by E et al. [1] and Abdulle et al. [2] . In particular, we focus on the specific case where the sizes of the heterogeneities are spread but not separated. We thus do not fall in the framework of the coarse graining method (see e.g. Geers et al. [3]) which requires proper scale separations. In the situation under consideration, the size of the smaller heterogeneities requires a fine discretization to capture the fluctuations of the fields, whereas the size of the larger heterogeneities imposes to define a large Representative Volume Element (RVE).

Various authors have greatly contributed to propose two step numerical homogenization methods to overcome the high computational and memory costs a direct refined simulation would require. Examples are the Multiscale Finite Elements Method [4, 5] and grid blocks or moving window techniques [6-13]. To

\footnotetext{
*Corresponding author. Tel.: +33 164153749

Email addresses: francois.bignonnet@enpc.fr (François Bignonnet), karam.sab@enpc.fr (Karam Sab), luc.dormieux@enpc.fr (Luc Dormieux), sebastien.brisard@ifsttar.fr (Sébastien Brisard)
} 
prevent possible confusions, the following terminology will be adopted in the remainder of this article (see also Fig. 1). Micro-scale heterogeneous elastic properties are upscaled by the resolution of a homogenization problem to derive homogenized elastic properties at the macroscopic scale. The common point of all the above mentioned methods is to consider two levels of discretization of the homogenization problem: a coarse discretization to capture the long range fluctuations of the fields or structural effects and a fine discretization to integrate lower scale information. The coarse discretization is made of coarse elements whose size defines a mesoscopic scale, which is separated neither from the macroscopic nor the microscopic scales.

In the first step of the two step numerical homogenization methods, a local problem is defined on each coarse element to derive effective properties of the coarse element. The aim is to transfer fine scale information to the coarse scale by solving the local problems, which are decoupled. In the second step, the homogenization problem is solved using the coarse discretization and the effective properties derived from the first step.

In the literature, the solutions to the local problems are used to define either shape functions of the coarse elements in Multiscale Finite Elements Method or effective properties of the coarse element by averaging in grid blocks or moving window techniques. The effective properties intrinsically depends on the size of the coarse discretization: large sizes create a smoothing effect, while an undesired resonance effect arises when the coarse discretization size is of the order of the fluctuations of the fields.

A first issue is encountered in the definition of the local problem itself: specifically, the choice of boundary conditions is critical. A great variety of boundary conditions have been used. Periodic boundary conditions applied directly on the boundary of the coarse elements have been widely used [6, 7, 10-13]. Dirichlet, Neumann and periodic boundary conditions have been compared by Yue and E [10], who concluded the periodic boundary conditions perform better in general. Chen et al. [9] proposed to iterate coarse and fine discretizations resolutions, feeding the local problems with boundary conditions interpolated from the coarse discretization solution. In view of performing a conforming Galerkin discretization, Hou and Wu [4] considered, for each node of the coarse element, imposed displacement of 1 on one node and 0 on the others, with either linear or more complex interpolations between the nodes.

When the size of the coarse discretization is of the order of the length scale of the fluctuations of the fields, the error on the full solution to the homogenization problem is maximal. This is known as the resonance effect $[4,7]$, which is amplified when the boundary conditions are imposed directly on the boundary of the coarse element. According to $\mathrm{Wu}$ et al. [7], the resonance effect has two origins: the cell resonance effect which is linked to ratio of the coarse discretization size to the fluctuations of the microstructure, and the non-conformation of the boundary conditions applied to the different local problems. To reduce this second origin of the resonance effect, a popularly used correction is to practice oversampling $[4,5,7,8,10]$. Oversampling means considering an oversampling region around the coarse element in the local problem so as to apply the boundary conditions slightly away from the boundary of the coarse element. The typical thickness of the oversampling region is the size of the fluctuations of the fields [4, 7]. Other strategies to reduce boundary effects have been devised, such as the use of a smoothing filter [10] or truncation (also called erosion) $[10,14,15]$ of the local solutions in the averaging process. These two strategies provide more robust results with Dirichlet boundary conditions, but have minor effects with periodic boundary conditions $[10]$.

A second issue, encountered while oversampling, is the definition of the effective properties of the coarse elements. Since no scale separation is ensured in this micro-to-meso transition, the effective properties have neither material nor energetical basis. Their definition is thus somewhat arbitrary. Whenever classical Dirichlet, Neumann or periodic boundary conditions are applied directly at the boundary of the coarse element in the local problem, the volume averages of the strain and stress in the coarse element are related by a symmetric positive definite tensor [7, 14], which is used as an effective property of the coarse element. However, while oversampling or eroding, symmetry and positive definiteness are lost using such direct averaging [14]. Some authors overcome this situation either by enforcing symmetry of the coarse element effective tensor using least-square procedures [9] or by introducing a procedure equivalent to energy averaging $[14,15]$.

The purpose of this paper is to propose a framework of filtering the homogenization problem, within the framework of periodic homogenization in linear elasticity. As a by product, the filtering framework allows to 


\section{homogenization problem}

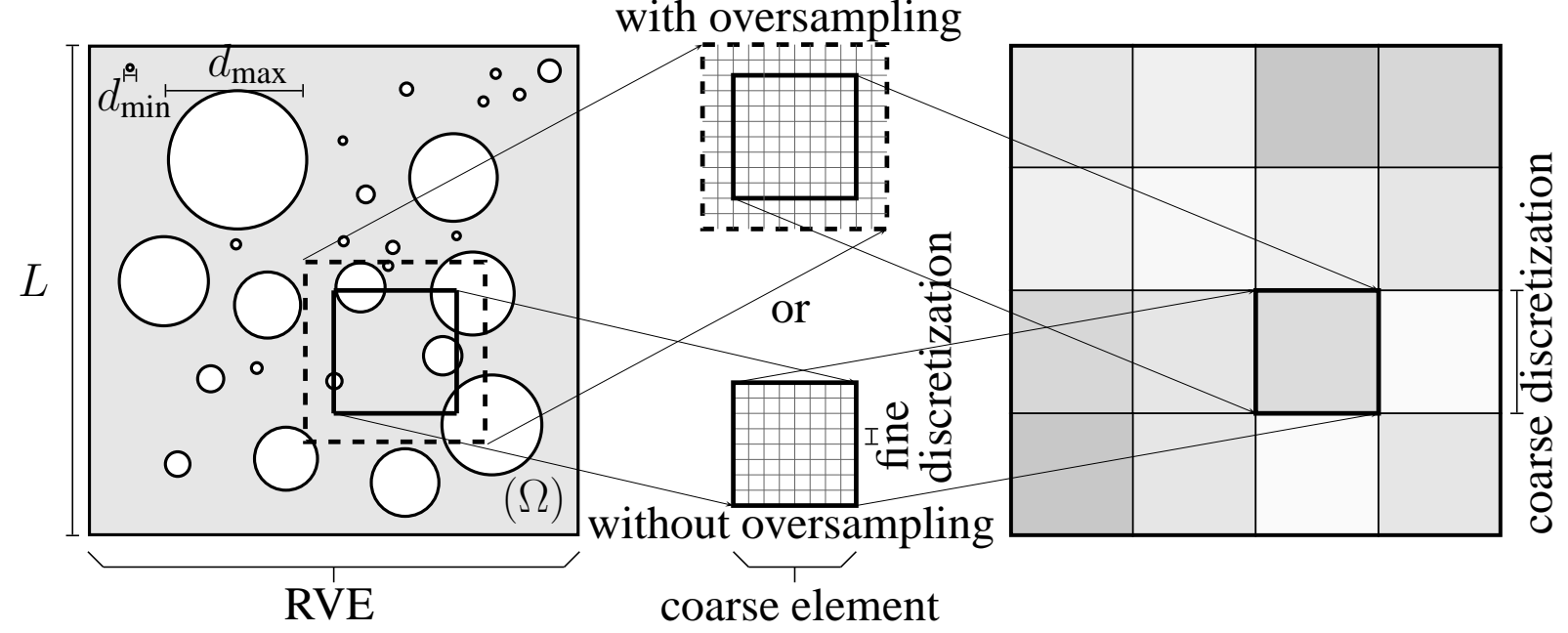

Figure 1: Classical two step numerical homogenization method. Left: homogenization problem defined on the Representative Volume Element (RVE). While $d_{\min }<d_{\max }$ are possibly distant but of the same order of magnitude, scale separation imposes $d_{\max } \ll L$. Center: local problem defined on a coarse element, with or without oversampling. A fine discretization is used to solve the local problem numerically. Right: coarse discretization of the homogenization problem with effective properties derived from the local problems.

address the two above mentioned issues: local problem boundary conditions and definition of the effective properties.

The paper is organized as follows. First, classical definitions and results on homogenization in periodic elasticity are recalled in section 2. Second, we present the new theoretical filtering framework in section 3 , defined on the undiscretized homogenization problem. For any microstructure to be homogenized, the filtering process produces a hierarchy of filtered problems, whose stiffness fields are smoother than the original one, but which have exactly the same homogenized stiffness. Additionally, two other types of filtered problems are derived, whose homogenized stiffnesses give close bounds on the original one. The properties of the filtered problems are then studied numerically in section 4. Next, a link is established in section 5 between filtered stiffness and coarse element effective stiffness concepts, with a specific focus on oversampling. Based on the findings of section 3, our proposed coarse effective stiffness definition differs from exisiting definitions based on filtered averaging. Convergence with oversampling region thickness of the effective stiffness to the filtered stiffness is studied numerically. Finally, section 6 revisits the existing two step numerical homogenization methods and associated sources of errors from the filtering point of view.

\section{Background}

\subsection{The homogenization problem in periodic elasticity}

To start with, some definitions and properties inherent to linear elastic periodic homogenization are recalled. An infinite domain occupying the whole space is considered with a periodic stiffness field $\mathbb{C}$, or equivalently a periodic compliance field $\mathbb{S}=\mathbb{C}^{-1}$. The periodicity allows to consider only a unit cell or Representative Volume Element (RVE) $\Omega$ of volume $|\Omega|$ (Fig. 1, left), which is periodically reproduced. Scale separation between the spatial fluctuations of $\mathbb{C}$ and the size of the RVE is assumed. The boundary $\partial \Omega$ of $\Omega$ has an outward normal vector denoted $\boldsymbol{n}$.

A macroscopic stress $\boldsymbol{\Sigma}$ results in a macroscopic strain $\boldsymbol{E}$ such that $\boldsymbol{\Sigma}=\mathbb{C}^{\text {hom }}: \boldsymbol{E}$ where $\mathbb{C}^{\text {hom }}$ is the homogenized stiffness tensor. The loading mode can be either an imposed macroscopic stress $\boldsymbol{\Sigma}$ or an imposed macroscopic strain $\boldsymbol{E}$. The inverse of $\mathbb{C}^{\text {hom }}$ is the homogenized compliance tensor $\mathbb{S}^{\text {hom }}$.

Within the framework of periodic homogenization, considered macroscopic loading modes imply periodic boundary conditions on $\Omega$. Namely, the displacement field $\boldsymbol{\xi}$ is the sum of a $\Omega$-periodic displacement $\boldsymbol{\xi}^{\text {per }}$ 
and a linear displacement $\boldsymbol{E} \cdot \boldsymbol{z}$, where $\boldsymbol{z}$ is the spatial coordinate. The associated strain field $\boldsymbol{\epsilon}=\operatorname{grad}^{s}(\boldsymbol{\xi})$, where $\operatorname{grad}^{s}$ denotes the symmetric part of the gradient, is $\Omega$-periodic. The average strain is $\overline{\boldsymbol{\epsilon}}=\boldsymbol{E}$, where the volume average of a field $f$ over the RVE is noted $\bar{f}$. The stress field $\boldsymbol{\sigma}$ is $\Omega$-periodic, so that periodic boundary conditions additionally require that $\boldsymbol{\sigma} \cdot \boldsymbol{n}$ is anti-periodic on $\partial \Omega$. Considered strain and stress fields are assumed square-integrable over $\Omega$. The fields are defined on the whole space, but periodicity of the microstructure allows to focus on the following definitions.

The set $\mathcal{K}(\boldsymbol{E})$ of kinematically admissible strain fields with the macroscopic strain $\boldsymbol{E}$ is defined as

$$
\mathcal{K}(\boldsymbol{E})=\left\{\boldsymbol{\epsilon}^{\prime}=\operatorname{grad}^{s}\left(\boldsymbol{\xi}^{\prime}\right) \mid \boldsymbol{\epsilon}^{\prime} \text { is } \Omega \text {-periodic, } \overline{\boldsymbol{\epsilon}^{\prime}}=\boldsymbol{E}\right\} .
$$

The set $\mathcal{K}$ is the union of all the sets $\mathcal{K}(\boldsymbol{E})$ for any $\boldsymbol{E}$. An element of $\mathcal{K}$ is called a kinematically admissible strain field.

Dually, the set $\mathcal{S}(\boldsymbol{\Sigma})$ of statically admissible stress fields with the macroscopic stress $\boldsymbol{\Sigma}$ is defined as

$$
\mathcal{S}(\boldsymbol{\Sigma})=\left\{\boldsymbol{\sigma}^{\prime} \mid \operatorname{div}\left(\boldsymbol{\sigma}^{\prime}\right)=\mathbf{0}, \boldsymbol{\sigma}^{\prime} \text { is } \Omega \text {-periodic, } \overline{\boldsymbol{\sigma}^{\prime}}=\boldsymbol{\Sigma}\right\} .
$$

The set $\mathcal{S}$ is the union of all the sets $\mathcal{S}(\boldsymbol{\Sigma})$ for any $\boldsymbol{\Sigma}$. An element of $\mathcal{S}$ is called a statically admissible stress field.

Making use of the properties of $\mathcal{K}(\boldsymbol{E})$ and $\mathcal{S}(\boldsymbol{\Sigma})$, Hill's lemma is derived

$$
\forall \boldsymbol{\epsilon}^{\prime} \in \mathcal{K}(\boldsymbol{E}) \text { and } \boldsymbol{\sigma}^{\prime} \in \mathcal{S}(\boldsymbol{\Sigma}), \overline{\boldsymbol{\epsilon}^{\prime}: \boldsymbol{\sigma}^{\prime}}=\boldsymbol{E}: \boldsymbol{\Sigma} \text {. }
$$

The homogenization problem with imposed macroscopic strain $\boldsymbol{E}$ consists in finding the unique couple of strain field $\boldsymbol{\epsilon}$ in $\mathcal{K}(\boldsymbol{E})$ and stress field $\boldsymbol{\sigma}$ in $\mathcal{S}$ such that the constitutive law $\boldsymbol{\sigma}=\mathbb{C}: \boldsymbol{\epsilon}$ is met at any point of the medium. Similarly, the homogenization problem with imposed macroscopic stress $\boldsymbol{\Sigma}$ is to find the unique couple of strain field $\boldsymbol{\epsilon}$ in $\mathcal{K}$ and stress field $\boldsymbol{\sigma}$ in $\mathcal{S}(\boldsymbol{\Sigma})$ such that $\boldsymbol{\sigma}=\mathbb{C}: \boldsymbol{\epsilon}$. To sum up, the initial homogenization problem is defined as

$$
\text { find } \boldsymbol{\epsilon} \in \mathcal{K}(\boldsymbol{E}) \text { and } \boldsymbol{\sigma} \in \mathcal{S} \text { such that } \boldsymbol{\sigma}(\boldsymbol{z})=\mathbb{C}(\boldsymbol{z}): \boldsymbol{\epsilon}(\boldsymbol{z}) \text { for all } \boldsymbol{z} \in \mathbb{R}^{3}
$$

or, equivalently

$$
\text { find } \boldsymbol{\sigma} \in \mathcal{S}(\boldsymbol{\Sigma}) \text { and } \boldsymbol{\epsilon} \in \mathcal{K} \text { such that } \boldsymbol{\sigma}(\boldsymbol{z})=\mathbb{C}(\boldsymbol{z}): \boldsymbol{\epsilon}(\boldsymbol{z}) \text { for all } \boldsymbol{z} \in \mathbb{R}^{3}
$$

\subsection{The homogenized stiffness tensor}

When a macroscopic strain $\boldsymbol{E}$ is imposed, the solution to the homogenization problem is linear in $\boldsymbol{E}$. Thus, the strain field may be formally written $\boldsymbol{\epsilon}(\boldsymbol{z})=\mathbb{A}(\boldsymbol{z}): \boldsymbol{E}$, where $\mathbb{A}$ is the fourth-order strain localization tensor. Then, the homogenized stiffness of the RVE is $\mathbb{C}^{\text {hom }}=\overline{\mathbb{C}: \mathbb{A}}$. Alternatively, an energetic approach yields $\mathbb{C}^{\text {hom }}=\overline{{ }^{t} \mathbb{A}: \mathbb{C}: \mathbb{A}}$, where ${ }^{t} \mathbb{A}$ is the transpose of $\mathbb{A}$. Both expressions coincide by virtue of Hill's lemma.

Similarly, when a macroscopic stress $\boldsymbol{\Sigma}$ is imposed, the stress field may be formally written $\boldsymbol{\sigma}(\boldsymbol{z})=\mathbb{B}(\boldsymbol{z})$ : $\boldsymbol{\Sigma}$, where $\mathbb{B}$ is the fourth-order stress localization tensor. Then, the homogenized compliance of the RVE is $\mathbb{S}^{\text {hom }}=\overline{\mathbb{S}: \mathbb{B}}=\overline{{ }^{t} \mathbb{B}: \mathbb{S}: \mathbb{B}}$.

Because of the use of periodic boundary conditions, $\mathbb{S}^{\text {hom }}=\left(\mathbb{C}^{\text {hom }}\right)^{-1}$.

\subsection{Variational framework}

While a macroscopic strain $\boldsymbol{E}$ is imposed, the minimum of the potential energy principle is derived, stating the macroscopic strain energy density is minimum among all kinematically admissible fields $\boldsymbol{\epsilon}^{\prime} \in \mathcal{K}(\boldsymbol{E})$ at the solution $\boldsymbol{\epsilon}$ of the minimization problem

$$
\frac{1}{2} \boldsymbol{E}: \mathbb{C}^{\text {hom }}: \boldsymbol{E}=\frac{1}{2} \overline{\boldsymbol{\epsilon}: \mathbb{C}: \boldsymbol{\epsilon}}=\min _{\boldsymbol{\epsilon}^{\prime} \in \mathcal{K}(\boldsymbol{E})} \frac{1}{2} \overline{\boldsymbol{\epsilon}^{\prime}: \mathbb{C}: \boldsymbol{\epsilon}^{\prime}}
$$

Dually, while a macroscopic stress $\boldsymbol{\Sigma}$ is imposed, the minimum of the complementary energy principle is derived, stating the macroscopic stress energy density is minimum among all statically admissible fields $\boldsymbol{\sigma}^{\prime} \in \mathcal{S}(\boldsymbol{\Sigma})$ at the solution $\boldsymbol{\sigma}$ of the minimization problem

$$
\frac{1}{2} \boldsymbol{\Sigma}: \mathbb{S}^{\text {hom }}: \boldsymbol{\Sigma}=\frac{1}{2} \overline{\boldsymbol{\sigma}: \mathbb{S}: \boldsymbol{\sigma}}=\min _{\boldsymbol{\sigma}^{\prime} \in \mathcal{S}(\boldsymbol{\Sigma})} \frac{1}{2} \overline{\boldsymbol{\sigma}^{\prime}: \mathbb{S}: \boldsymbol{\sigma}^{\prime}}
$$




\section{Hierarchy of filtered heterogeneous media}

\subsection{Filtering process}

In this section, the solution to the initial homogenization problem (4) is supposed known. Macroscopically equivalent but locally smoother homogenization problems are sought by filtering the solution displacement and stress fields. The idea of filtering the problem by performing averages within moving windows has been considered by Baxter and Graham [11], Graham-Brady et al. [12], Acton and Graham-Brady [13]. Unlike the Generalized Method of Cell proposed by these authors, the filtering we propose is not defined based on the solution to the local problems, but rather on the solution to the whole homogenization problem, which was referred to as the brute-force Finite Element method in Graham-Brady et al. [12]. The following developments propose a generalization of the filtering concept and demonstrate new properties of the filtered problems within the framework of periodic homogenization.

The filtering process is based on a scalar mass function $\rho$ defined on the infinite space $\mathbb{R}^{3}$ such that $\int_{\mathbb{R}^{3}} \rho(\boldsymbol{z}) d V_{\boldsymbol{z}}=1$, as in $[5,10,16]$. A typical example is $\rho(\boldsymbol{z})=1 /|\mathcal{D}| \chi_{\mathcal{D}}$ where $\chi_{\mathcal{D}}$ is the characteristic function of the sliding window $\mathcal{D}[12]$.

Let us consider the convolution of a function $f$ with $\rho$, denoted $f^{*}$ and defined as for any point $\boldsymbol{z}$ of the infinite space $\mathbb{R}^{3}$ as

$$
f^{*}(\boldsymbol{z})=(\rho * f)(\boldsymbol{z})=\int_{\mathbb{R}^{3}} \rho(\boldsymbol{z}-\boldsymbol{y}) f(\boldsymbol{y}) d V_{y}
$$

The filtered function $f^{*}$ has the following properties

1. periodicity conservation: if $f$ is periodic on $\partial \Omega$, then $f^{*}$ too,

2. volume average conservation: $\bar{f}=\bar{f}^{*}$,

3. regularity transmission: $f^{*}$ benefits from the best regularity properties among $f$ and $\rho$.

4. derivation: $\frac{\partial f^{*}}{\partial z_{i}}(\boldsymbol{z})=\left(\frac{\partial f}{\partial z_{i}}\right)^{*}(\boldsymbol{z})$.

From these properties, the filtered fields naturally verify

$$
\begin{aligned}
& \boldsymbol{\sigma}^{\prime} \in \mathcal{S}(\boldsymbol{\Sigma}) \Rightarrow \boldsymbol{\sigma}^{*} \in \mathcal{S}(\boldsymbol{\Sigma}), \\
& \boldsymbol{\epsilon}^{\prime} \in \mathcal{K}(\boldsymbol{E}) \Rightarrow \boldsymbol{\epsilon}^{\prime *} \in \mathcal{K}(\boldsymbol{E}),
\end{aligned}
$$

from which it results that $\boldsymbol{\sigma}^{*}=\mathbb{B}^{*}: \boldsymbol{\Sigma} \in \mathcal{S}(\boldsymbol{\Sigma})$ for all $\boldsymbol{\Sigma}$ and $\boldsymbol{\epsilon}^{*}=\mathbb{A}^{*}: \boldsymbol{E} \in \mathcal{K}(\boldsymbol{E})$ for all $\boldsymbol{E}$.

\subsection{Macroscopically consistent definitions of the non-local stiffness}

The strain field $\boldsymbol{\epsilon}$ and stress field $\boldsymbol{\sigma}$ solutions to problem (4a) are filtered. Based on the results of section 3.1, $\boldsymbol{\epsilon}^{*}$ is kinematically admissible with the macroscopic strain $\boldsymbol{E}$ and $\boldsymbol{\sigma}^{*}$ is statically admissible. We then seek to which new homogenization problem are $\boldsymbol{\epsilon}^{*}$ and $\boldsymbol{\sigma}^{*}$ solution.

The new behavior law must be defined by the tensor which links $\boldsymbol{\sigma}^{*}$ to $\boldsymbol{\epsilon}^{*}$. Using the relations $\boldsymbol{\epsilon}^{*}=\mathbb{A}^{*}: \boldsymbol{E}$, $\boldsymbol{\sigma}^{*}=(\mathbb{C}: \mathbb{A})^{*}: \boldsymbol{E}$ and assuming that $\mathbb{A}^{*}$ is invertible, the sought relation writes $\boldsymbol{\sigma}^{*}=(\mathbb{C}: \mathbb{A})^{*}:\left(\mathbb{A}^{*}\right)^{-1}: \boldsymbol{\epsilon}^{*}$. Thus a consistent filtered stiffness field $\widetilde{\mathbb{C}}^{\mathrm{c}}$ is defined such that $\boldsymbol{\sigma}^{*}=\widetilde{\mathbb{C}}^{\mathrm{c}}: \boldsymbol{\epsilon}^{*}$, where

$$
\widetilde{\mathbb{C}}^{\mathrm{C}}=(\mathbb{C}: \mathbb{A})^{*}:\left(\mathbb{A}^{*}\right)^{-1} .
$$

The stiffness $\widetilde{\mathbb{C}}^{c}$ has minor symmetries but no major symmetry, that is $\widetilde{\mathbb{C}}_{i j k l}^{c}=\widetilde{\mathbb{C}}_{j i k l}^{c}=\widetilde{\mathbb{C}}_{i j l k}^{c} \neq \widetilde{\mathbb{C}}_{k l i j}^{c}$. Indeed, it neither has energetical basis nor benefits from boundary conditions relations. The non-symmetry is believed to be the signature of the non-local nature of this quantity.

Nonetheless, a consistent filtered linear problem $\left(\tilde{P}^{\mathrm{c}}\right)$ is defined as

$$
\text { find } \boldsymbol{\epsilon}^{\mathrm{c}} \in \mathcal{K}(\boldsymbol{E}) \text { and } \boldsymbol{\sigma}^{\mathrm{c}} \in \mathcal{S} \text { such that } \boldsymbol{\sigma}^{\mathrm{c}}(\boldsymbol{z})=\widetilde{\mathbb{C}}^{\mathrm{c}}(\boldsymbol{z}): \boldsymbol{\epsilon}^{\mathrm{c}}(\boldsymbol{z}) \text { for all } \boldsymbol{z} \in \mathbb{R}^{3} \text {. }
$$


We assume the solution of problem $\left(\tilde{P}^{\mathrm{c}}\right)$ to be unique. The problem $\left(\tilde{P}^{\mathrm{c}}\right)$ leads to the homogenized stiffness $\widetilde{\mathbb{C}}^{\mathrm{c}}$, hom, whose direct definition is such that $\overline{\boldsymbol{\sigma}^{\mathrm{c}}}=\widetilde{\mathbb{C}}^{\mathrm{c}}$, hom $: \boldsymbol{E}$. A priori, $\widetilde{\mathbb{C}}^{\mathrm{c}}$, hom may be non symmetric.

However, as previously stated, the filtered couple $\left(\boldsymbol{\epsilon}^{*}, \boldsymbol{\sigma}^{*}\right)$ is in $\mathcal{K}(\boldsymbol{E}) \times \mathcal{S}(\boldsymbol{\Sigma})$. Additionally, the filtered couple meets the constitutive equation $\sigma^{*}=\widetilde{\mathbb{C}}^{c}: \epsilon^{*}$ by definition. Thus, $\left(\boldsymbol{\epsilon}^{*}, \boldsymbol{\sigma}^{*}\right)$ is solution to the homogenization problem $\left(\tilde{P}^{c}\right)$. Assuming the problem $\left(\tilde{P}^{\mathrm{c}}\right)$ has a unique solution, $\overline{\boldsymbol{\sigma}}^{\mathrm{c}}=\overline{\boldsymbol{\sigma}^{*}}=\boldsymbol{\Sigma}$, so that the consistent filtered homogenization problem $\left(\tilde{P}^{\mathrm{c}}\right)$ leads to the homogenized stiffness of the original problem: $\widetilde{\mathbb{C}}^{\mathrm{c}, \text { hom }}=\mathbb{C}^{\text {hom }}$.

Alternatively, the application of Hill's lemma to the couple $\left(\boldsymbol{\epsilon}^{*}, \boldsymbol{\sigma}^{*}\right)$ gives

$$
\frac{1}{2} \overline{\epsilon^{*}: \widetilde{\mathbb{C}}^{\mathrm{c}}: \boldsymbol{\epsilon}^{*}}=\frac{1}{2} \overline{\boldsymbol{\sigma}^{*}: \boldsymbol{\epsilon}^{*}}=\frac{1}{2} \boldsymbol{\Sigma}: \boldsymbol{E}=\frac{1}{2} \boldsymbol{E}: \mathbb{C}^{\mathrm{hom}}: \boldsymbol{E},
$$

so that the direct and energetical definitions of $\widetilde{\mathbb{C}}^{c}$, hom coincide: $\widetilde{\mathbb{C}}^{c}$, hom $=\overline{\widetilde{\mathbb{C}}^{c}: \mathbb{A}^{c}}=\overline{{ }^{t} \mathbb{A}^{c}: \widetilde{\mathbb{C}}^{c}: \mathbb{A}^{c}}$, where $\mathbb{A}^{c}$ is the strain localization tensor of problem $\left(\tilde{P}^{\mathrm{c}}\right)$. Hence, the homogenization problem $\left(\tilde{P}^{\mathrm{c}}\right)$ leads, for any considered mass function $\rho$, to the homogenized stiffness of the original problem, that is

$$
\widetilde{\mathbb{C}}^{\mathrm{c}, \text { hom }}=\mathbb{C}^{\text {hom }} .
$$

The above property will be referred to as the property of consistency in the remainder of this paper.

Similarly, considering the stress concentration tensor $\mathbb{B}$ yields the definition of an analogous $\widetilde{\mathbb{S}}^{\mathrm{c}}$ which is exactly the inverse of $\widetilde{\mathbb{C}}^{\mathrm{c}}$, that is, assuming $\mathbb{B}^{*}$ is invertible, $\widetilde{\mathbb{S}}^{c}=(\mathbb{S}: \mathbb{B})^{*}:\left(\mathbb{B}^{*}\right)^{-1}$.

To overcome the questions arising from the non symmetry of $\widetilde{\mathbb{C}}^{c}$, two alternative definitions are studied.

\subsection{Other, non-consistent, definitions of the non-local stiffness}

\subsubsection{Kinematic approach}

The local strain elastic energy density is $w^{\mathrm{k}}=\frac{1}{2} \boldsymbol{\epsilon}: \mathbb{C}: \boldsymbol{\epsilon}$. Let us consider the filtered energy $w^{\mathrm{k} *}$ of the initial homogenization problem (4) with imposed macroscopic strain $\boldsymbol{E}$. From prop. 2 of the filtering process, $\overline{w^{\mathrm{k} *}}=\overline{w^{\mathrm{k}}}=\frac{1}{2} \boldsymbol{E}: \mathbb{C}^{\text {hom }}: \boldsymbol{E}$. Using the results of section 3.1, the filtered strain energy may be rewritten

$$
\begin{aligned}
w^{\mathrm{k} *} & =\frac{1}{2}(\boldsymbol{\epsilon}: \mathbb{C}: \boldsymbol{\epsilon})^{*}=\frac{1}{2} \boldsymbol{E}:\left({ }^{t} \mathbb{A}: \mathbb{C}: \mathbb{A}\right)^{*}: \boldsymbol{E} \\
& =\frac{1}{2} \epsilon^{*}:{ }^{t}\left(\mathbb{A}^{*}\right)^{-1}:\left({ }^{t} \mathbb{A}: \mathbb{C}: \mathbb{A}\right)^{*}:\left(\mathbb{A}^{*}\right)^{-1}: \boldsymbol{\epsilon}^{*}=\frac{1}{2} \epsilon^{*}: \widetilde{\mathbb{C}}^{\mathrm{k}}: \boldsymbol{\epsilon}^{*},
\end{aligned}
$$

where the positive definite stiffness $\widetilde{\mathbb{C}}^{\mathrm{k}}$ ( $\mathrm{k}$ stands for kinematic) is defined as

$$
\widetilde{\mathbb{C}}^{\mathrm{k}}={ }^{t}\left(\mathbb{A}^{*}\right)^{-1}:\left({ }^{t} \mathbb{A}: \mathbb{C}: \mathbb{A}\right)^{*}:\left(\mathbb{A}^{*}\right)^{-1} .
$$

The problem with this strain energy definition of $\widetilde{\mathbb{C}}^{\mathrm{k}}$ is that $\widetilde{\mathbb{C}}^{\mathrm{k}}: \epsilon^{*}$ is not statically admissible in the general case, since the divergence equation may not be met.

Let us consider a new filtered problem $\left(\tilde{P}^{\mathrm{k}}\right)$ defined as

$$
\text { find } \boldsymbol{\epsilon}^{\mathrm{k}} \in \mathcal{K}(\boldsymbol{E}) \text { and } \boldsymbol{\sigma}^{\mathrm{k}} \in \mathcal{S} \text { such that } \boldsymbol{\sigma}^{\mathrm{k}}(\boldsymbol{z})=\widetilde{\mathbb{C}}^{\mathrm{k}}(\boldsymbol{z}): \boldsymbol{\epsilon}^{\mathrm{k}}(\boldsymbol{z}) \text { for all } \boldsymbol{z} \in \mathbb{R}^{3} \text {. }
$$

The homogenized stiffness $\widetilde{\mathbb{C}}^{\mathrm{k}, \text { hom }}$ associated to problem $\left(\tilde{P}^{\mathrm{k}}\right)$ is defined such that $\overline{\boldsymbol{\epsilon}^{\mathrm{k}}: \widetilde{\mathbb{C}}^{\mathrm{k}}: \boldsymbol{\epsilon}^{\mathrm{k}}}=\boldsymbol{E}: \widetilde{\mathbb{C}}^{\mathrm{k}, \text { hom }}$ : E.

Since $\widetilde{\mathbb{C}}^{\mathrm{k}}: \boldsymbol{\epsilon}^{*}$ is not statically admissible, the couple $\left(\boldsymbol{\epsilon}^{*}, \widetilde{\mathbb{C}}^{\mathrm{k}}: \boldsymbol{\epsilon}^{*}\right)$ differs from the actual solution $\left(\boldsymbol{\epsilon}^{\mathrm{k}}, \boldsymbol{\sigma}^{\mathrm{k}}\right)$ to $\left(\tilde{P}^{\mathrm{k}}\right)$. The solution strain field $\boldsymbol{\epsilon}^{\mathrm{k}}$ minimizes the potential energy $\overline{\frac{1}{2}} \overline{\boldsymbol{\epsilon}^{\prime}: \widetilde{\mathbb{C}}^{\mathrm{k}}: \boldsymbol{\epsilon}^{\prime}}$ in $\mathcal{K}(\boldsymbol{E})$. Since $\boldsymbol{\epsilon}^{*}$ is in $\mathcal{K}(\boldsymbol{E})$ however, solving $\left(\tilde{P}^{\mathrm{k}}\right)$, a lower bound is obtained

$$
\frac{1}{2} \boldsymbol{E}: \widetilde{\mathbb{C}}^{\mathrm{k}, \mathrm{hom}}: \boldsymbol{E}=\frac{1}{2} \overline{\epsilon^{\mathrm{k}}: \widetilde{\mathbb{C}}^{\mathrm{k}}: \boldsymbol{\epsilon}^{\mathrm{k}}} \leqslant \frac{1}{2} \overline{\epsilon^{*}: \widetilde{\mathbb{C}}^{\mathrm{k}}: \boldsymbol{\epsilon}^{*}}=\frac{1}{2} \boldsymbol{E}: \mathbb{C}^{\mathrm{hom}}: \boldsymbol{E} .
$$




\subsubsection{Static approach}

The local stress elastic energy density is $w^{\mathrm{s}}=\frac{1}{2} \boldsymbol{\sigma}: \mathbb{S}: \boldsymbol{\sigma}$ (s stands for static). Using the results of section 3.3.1, the filtered energy $w^{s *}$ may be rewritten

$$
w^{s *}=\frac{1}{2} \sigma^{*}: \widetilde{\mathbb{S}}^{s}: \sigma^{*}
$$

where the positive definite compliance $\widetilde{\mathbb{S}}^{\mathrm{s}}=\left(\widetilde{\mathbb{C}}^{\mathrm{s}}\right)^{-1}$ is defined as

$$
\widetilde{\mathbb{S}}^{\mathrm{s}}={ }^{t}\left(\mathbb{B}^{*}\right)^{-1}:\left({ }^{t} \mathbb{B}: \mathbb{S}: \mathbb{B}\right)^{*}:\left(\mathbb{B}^{*}\right)^{-1} .
$$

Note that alternatively, the following equivalent expression may be derived

$$
\widetilde{\mathbb{S}}^{\mathrm{s}}={ }^{t}\left((\mathbb{C}: \mathbb{A})^{*}\right)^{-1}:\left({ }^{t} \mathbb{A}: \mathbb{C}: \mathbb{A}\right)^{*}:\left((\mathbb{C}: \mathbb{A})^{*}\right)^{-1} .
$$

The problem with this stress energy definition of $\widetilde{\mathbb{S}}^{s}$ is that $\widetilde{\mathbb{S}}^{s}: \sigma^{*}$ is not kinematically admissible in the general case since it may not derive from a displacement field.

Let us consider another filtered problem $\left(\tilde{P}^{\mathrm{s}}\right)$ defined as

$$
\text { find } \boldsymbol{\epsilon}^{\mathrm{s}} \in \mathcal{K}(\boldsymbol{E}) \text { and } \boldsymbol{\sigma}^{\mathrm{s}} \in \mathcal{S} \text { such that } \boldsymbol{\sigma}^{\mathrm{s}}(\boldsymbol{z})=\widetilde{\mathbb{C}}^{\mathrm{s}}(\boldsymbol{z}): \boldsymbol{\epsilon}^{\mathrm{s}}(\boldsymbol{z}) \text { for all } \boldsymbol{z} \in \mathbb{R}^{3} \text {. }
$$

The homogenized compliance $\widetilde{\mathbb{S}}^{\mathrm{s}}$, hom associated to problem $\left(\tilde{P}^{\mathrm{s}}\right)$ is defined such that $\overline{\boldsymbol{\sigma}^{\mathrm{s}}: \widetilde{\mathbb{S}}^{\mathrm{s}}: \boldsymbol{\sigma}^{\mathrm{s}}}=\boldsymbol{\Sigma}$ : $\widetilde{\mathbb{S}}^{\mathrm{s}}$, hom $: \boldsymbol{\Sigma}$. The inverse of $\widetilde{\mathbb{S}}^{\mathrm{s}}$, hom is the homogenized stiffness $\widetilde{\mathbb{C}}^{\mathrm{s}}$, hom $=\left(\widetilde{\mathbb{S}}^{\mathrm{s}}, \text { hom }\right)^{-1}$.

Since $\widetilde{\mathbb{S}}^{\mathrm{s}}: \boldsymbol{\sigma}^{*}$ is not kinematically admissible, it differs from the actual solution $\left(\boldsymbol{\epsilon}^{\mathrm{s}}, \boldsymbol{\sigma}^{\mathrm{s}}\right)$ to $\left(\tilde{P}^{\mathrm{s}}\right)$. The stress field $\boldsymbol{\sigma}^{\mathrm{s}}$ minimizes the complementary energy $\frac{1}{2} \overline{\sigma^{\prime}: \widetilde{\mathbb{S}}^{s}: \boldsymbol{\sigma}^{\prime}}$ in $\mathcal{S}(\Sigma)$. Since $\boldsymbol{\sigma}^{*}$ is in $\mathcal{S}(\Sigma)$, the following inequality is verified

$$
\frac{1}{2} \boldsymbol{\Sigma}: \widetilde{\mathbb{S}}^{\mathrm{s}}, \text { hom }: \boldsymbol{\Sigma}=\frac{1}{2} \overline{\boldsymbol{\sigma}^{\mathrm{s}}: \widetilde{\mathbb{S}}^{\mathrm{s}}: \boldsymbol{\sigma}^{\mathrm{s}}} \leqslant \frac{1}{2} \overline{\boldsymbol{\sigma}^{*}: \widetilde{\mathbb{S}}^{\mathrm{s}}: \boldsymbol{\sigma}^{*}}=\frac{1}{2} \boldsymbol{\Sigma}: \mathbb{S}^{\text {hom }}: \boldsymbol{\Sigma} .
$$

Finally the kinematic and static energy definitions of the non-local behavior lead to the following upper and lower bounds, where the inequalities must be understood in the sense of associated quadratic forms:

$$
\widetilde{\mathbb{C}}^{\mathrm{k}, \text { hom }} \leq \mathbb{C}^{\text {hom }}=\widetilde{\mathbb{C}}^{\mathrm{c}, \text { hom }} \leq \widetilde{\mathbb{C}}^{\mathrm{s}, \text { hom }} .
$$

\section{Numerical study of filtered problems}

The aim of this section is to verify numerically the consistency property (12) established in section 3.2 as well as the bounds (22) resulting from the energy approach of section 3.3.

\subsection{Numerical filtering and resolution of a non symmetric linear elasticity homogenization problem}

Throughout the paper, numerical simulations are carried out using a regular discretization grid for the sake of simplicity and opportunity to use FFT-based homogenization methods. The confusion between elements and pixels (or voxels in 3D) will thus often be made. However, the concepts exposed previously are not restricted to FFT-based homogenization, but hold for any other numerical method, such as FEM.

FFT-based homogenization methods have been initially introduced by Moulinec and Suquet [17], and may be extended to the case where the stiffness is not symmetric (See Appendix A for details) to solve consistently filtered problems (10).

The determination of the filtered stiffness fields requires the strain or stress localization tensors. To this end, using imposed macroscopic strains for example, several loading modes are successively imposed on the microstructure. In two dimensions (plane strains), three linearly independent macroscopic strains $\boldsymbol{E}$ must be imposed, for instance: $\boldsymbol{E}=\boldsymbol{e}_{x} \otimes \boldsymbol{e}_{x}, \boldsymbol{e}_{y} \otimes \boldsymbol{e}_{y}$, and $\sqrt{2} / 2\left(\boldsymbol{e}_{x} \otimes \boldsymbol{e}_{y}+\boldsymbol{e}_{y} \otimes \boldsymbol{e}_{x}\right)$. In three dimensions, six linearly independent macroscopic strains $\boldsymbol{E}$ must be imposed. For each of these loading modes, the 
local strains and stresses are computed numerically by solving problem (4) with FFT-based methods. The components of the strain and stress fields for each unit loading mode characterize the strain and stress localization tensors fields. Each component of the localization tensors fields is then filtered efficiently by resorting to FFT to compute the convolution products. Then, the consistent, kinematic and static filtered stiffnesses are computed voxel by voxel by means of the definitions (9), (14) and (18) or (19) respectively.

Several discretization issues are encountered through the above process. These issues are discussed in the context of a numerical resolution by a FFT-based method. For this method, the discretization of a stiffness field $\mathbb{C}(\boldsymbol{z})$ in FFT-based methods has been discussed in [18]. For each voxel $\beta$ of the regular discretization grid, the discretized stiffness $\mathbb{C}_{\beta}$ is defined such that

$$
\left(\mathbb{C}_{\beta}-\mathbb{C}_{0}\right)^{-1}=\overline{\left(\mathbb{C}(\boldsymbol{z})-\mathbb{C}_{0}\right)^{-1}}{ }^{\beta}
$$

where $\mathbb{C}_{0}$ is a reference homogeneous stiffness used in the FFT-based method and ${ }^{-\beta}$ denotes the volume average over a given voxel $\beta$, .

First, the solution to the homogenization problem (4) is not known exactly, but approximately. More precisely, the output of the numerical resolution by a FFT-based method is an approximation of the volume average over each voxel of the strain field. Next, when the filter is applied to the numerical solutions, only approximations of the volume average over each voxel of the strain and stress concentration fields are retrieved. In other words, the filtering of the numerical solution provides only approximations of $\overline{\mathbb{A}}^{\beta}$ and ${\overline{(\mathbb{C}: \mathbb{A})^{*}}}^{\beta}$ for each voxel $\beta$ (provided $\mathbb{C}(\boldsymbol{z})$ is constant per voxel).

Consequently, the filtered stiffness field cannot be discretized as proposed in [18]. Instead, the following approximation has been made for the discretization of the filtered stiffness field $\widetilde{\mathbb{C}}^{c}$ :

$$
\widetilde{\mathbb{C}}_{\beta}^{c}=\mathbb{C}_{0}+\left(\overline{\left[(\mathbb{C}: \mathbb{A})^{*}:\left(\mathbb{A}^{*}\right)^{-1}-\mathbb{C}_{0}\right]^{-1}}\right)^{-1} \approx{\overline{(\mathbb{C}: \mathbb{A})^{*}}}^{\beta}:\left({\overline{\mathbb{A}^{*}}}^{\beta}\right)^{-1}
$$

and similarly for $\widetilde{\mathbb{C}}^{k}$ and $\widetilde{\mathbb{C}}^{s}$. This approximation is deemed reasonable in the most likely case where $\widetilde{\mathbb{C}}$ is a continuous function which varies slowly as compared to the discretization.

Second, the size of the discretization of the filtered problems must be chosen. The filtered stiffness fields have been introduced so that the length scale of the spatial fluctuations is greater for $\widetilde{\mathbb{C}}$ than for $\mathbb{C}$. The size of the discretization of the filtered problems will be studied numerically in Sec. 4.4.

\subsection{Effect of filter size and shape on the filtered stiffness field}

Two types of filters have been considered (Fig. 2):

- Square or cubic window filter: defined as zero outside a square or cube of size $H$ centered at the origin and constant inside so that its average is 1 (Fig. 2(a)). The square filter is typical of moving window schemes [11-13], except the boundary conditions are not applied at the boundary of the window. This filter has preferential orientations along the axes and sharp edges.

- Smooth gaussian filter: specifically designed to be smooth in both direct and Fourier space with no preferential orientation (Fig. 2(b)). The gaussian filter is classically used in image analysis. A filtering algorithm with the smooth gaussian filter is efficiently implemented using Fast Fourier Transform (FFT) to compute the convolution product.

As an example of the filtering procedure, a microstructure made of cylindrical fibers embedded in a homogeneous matrix is considered. The volume fraction of the cylindrical fibers is $12 \%$, their diameter $d=32$ pixels and the discretization grid has $512^{2}$ pixels. Both phases are isotropic linear elastic. The matrix has a shear modulus 1 and the fibers have a shear modulus 100, while both have a Poisson's ratio of 0.2 , so that an elastic contrast of 100 is obtained.

Maps of the initial and consistent filtered stiffness field (component $\widetilde{C}_{1111}$ ) are shown in Fig. 3. The solution strain and stress fields necessary to the filtering process have been obtained by application of the 


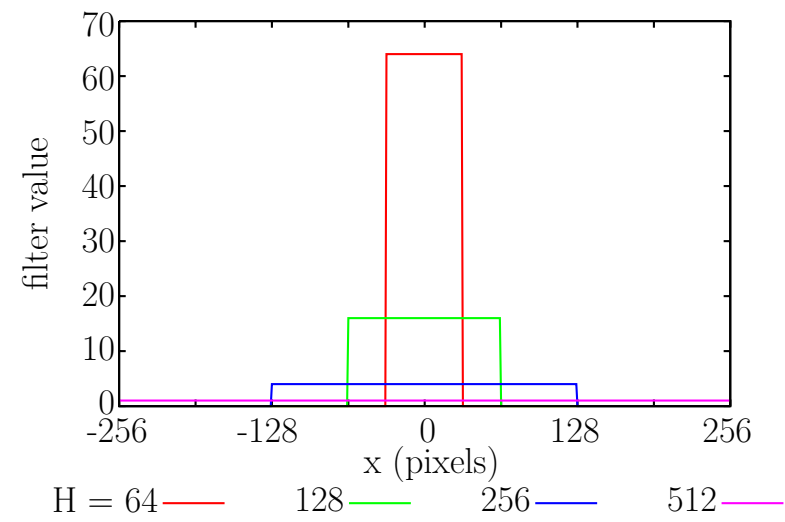

(a) square window filter

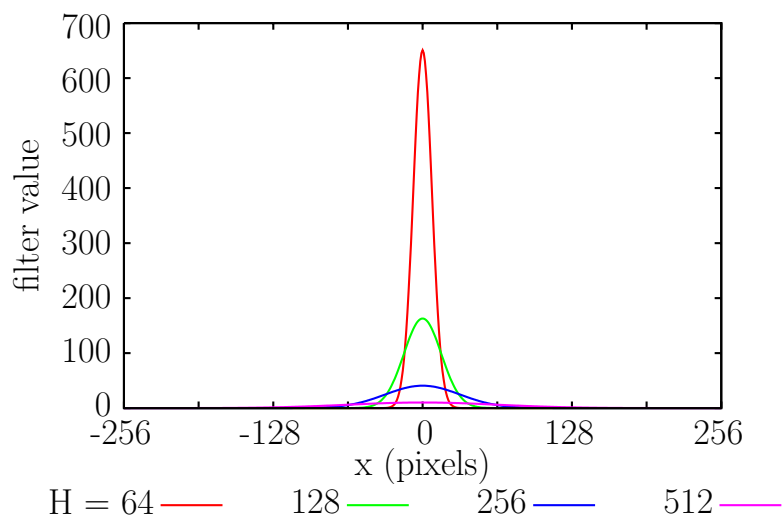

(b) smooth gaussian filter

Figure 2: Cut along the $\mathrm{x}$ axis of the square window and smooth gaussian filters on a $512^{2}$ pixels discretization grid, for different filter sizes.

FFT-based homogenization method described in Brisard and Dormieux [18]. The filter size $H$ is expressed in multiples of the fiber diameter $d$. Starting from small filter sizes, the first effect observed is a smoothing of the stiffness jump across the matrix-fiber interface, due to local averaging effect (Fig. 4(b)). When the filter size reaches about the fiber diameter, a sharp reduction in contrast is observed (Fig. 4(b)), as already noticed by Yue and E [10], Baxter and Graham [11]. In the mean time, the difference between the two filters becomes clearer: the anisotropy of the square window is transferred to the filtered stiffness, square patterns being observed around the fibers (Fig. 3). For filter sizes above a few fiber diameters, the contrast is very low.

However, the square window filter has no regularization property: some discontinuities of the filtered stiffness field are observed (Fig. 4(b)).

This example highlights that the smoothing effect due to filtering has two distinct origins:

1. increasing filter size creates an (indirect) averaging effect of the stiffness field,

2 . by virtue of the convolution product, regularity properties of the filter are transferred to the filtered stiffness field.

Similar trends have been observed for the non consistent filtered stiffness fields $\widetilde{\mathbb{C}}^{\mathrm{k}}$ and $\widetilde{\mathbb{C}}^{\mathrm{s}}$, which are close from $\widetilde{\mathbb{C}}^{c}$ in this specific case. However, when very high contrasts are encountered, the numerical solution fields suffer from discretization effect and the computed filtered stiffness may be ill behaved, especially around matrix-fiber interfaces. Namely, when the fibers are much softer than the matrix, the strain localization tensor is poorly approximated inside and just around the fibers, which may cause $\widetilde{\mathbb{C}}^{c}$ and $\widetilde{\mathbb{C}}^{\mathrm{k}}$ to diverge locally. In this case however, $\widetilde{\mathbb{C}}^{\mathrm{s}}$ is well behaved. On the contrary, when the fibers are much stiffer than the matrix, the stress localization tensor is poorly approximated inside and just around the fibers, which may cause $\widetilde{\mathbb{C}}^{\mathrm{c}}$ and $\widetilde{\mathbb{C}}^{\mathrm{s}}$ to oscillate inside the fibers, whereas $\widetilde{\mathbb{C}}^{\mathrm{k}}$ is well behaved. Thus, $\widetilde{\mathbb{C}}^{\mathrm{s}}$ could be used in the presence of voids and $\widetilde{\mathbb{C}}^{c}$ for rigid inclusions whereas the other combinations are not defined.

In turn, when complex microstructures are encountered (see e.g. Fig. 5(a)), the discretized filtered stiffness fields may diverge locally (see Fig. 5(c)) because of the inverse of the filtered strain or stress concentration tensor which appears in (9), (14) and (18). For this specific microstructure, $\widetilde{\mathbb{C}}^{\mathrm{c}}$ and $\widetilde{\mathbb{C}}^{\mathrm{k}}$ diverge locally whereas $\widetilde{\mathbb{C}}^{\mathrm{s}}$ is smooth (Fig. $5(\mathrm{~b})$ ). We have not been able to determine whether this diverging behavior arose as a consequence of an insufficient or unproper discretization of the numerical solution fields or are inherent to the filtered stiffness fields.

Nevertheless, even when the filtered stiffnesses happened to be ill behaved, the numerical inversion of the filtered localization tensor has always been observed to be possible. 


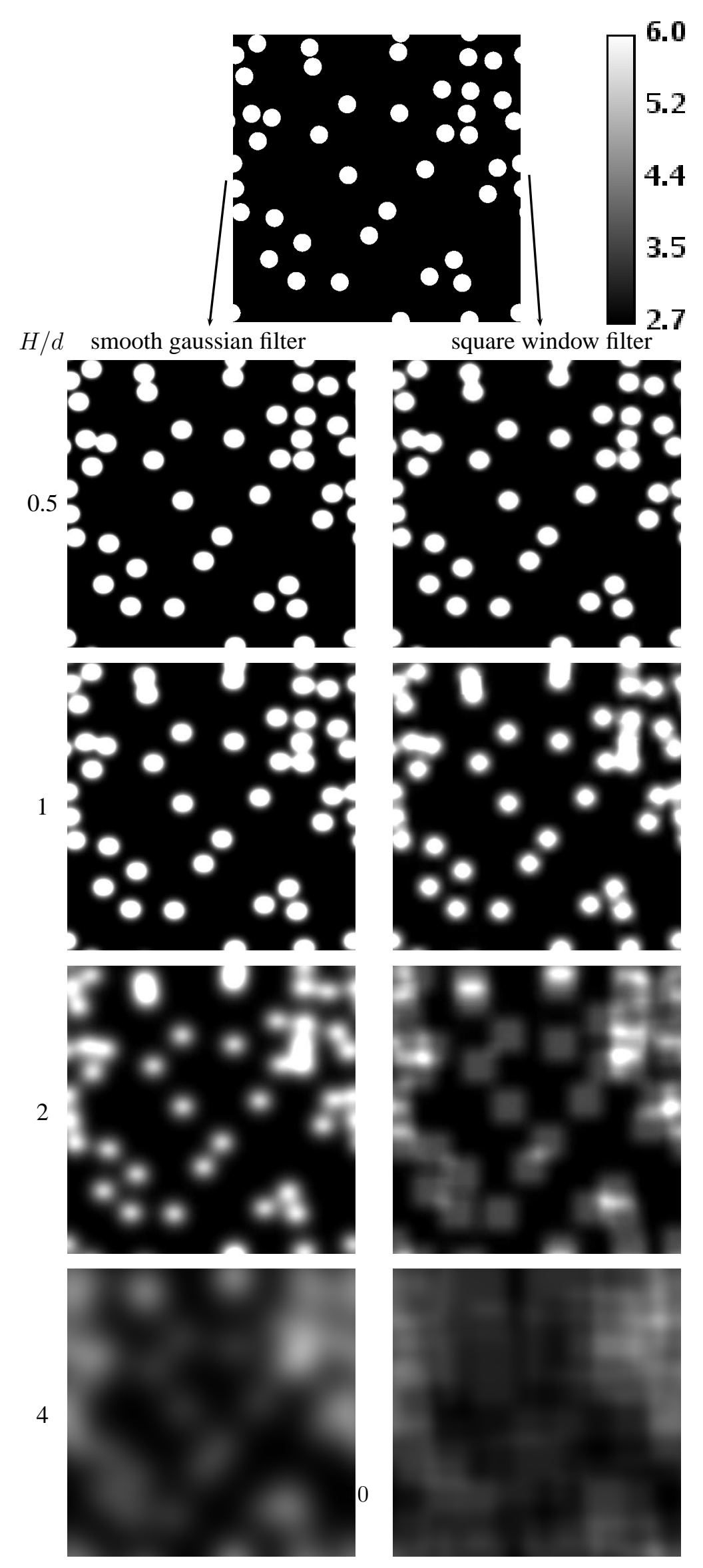

Figure 3: Comparison of consistent filtered stiffness component $\widetilde{C}_{1111}^{\mathrm{c}}$ maps for two kinds of filters and different sizes. The initial contrast is 100 , with $C_{1111}^{\text {matrix }}=2.67$ and $C_{1111}^{\text {fiber }}=267$, but the gray scale contrast has been adjusted to the range [2.7;6.0] for all filter sizes to better highlight the evolution. 


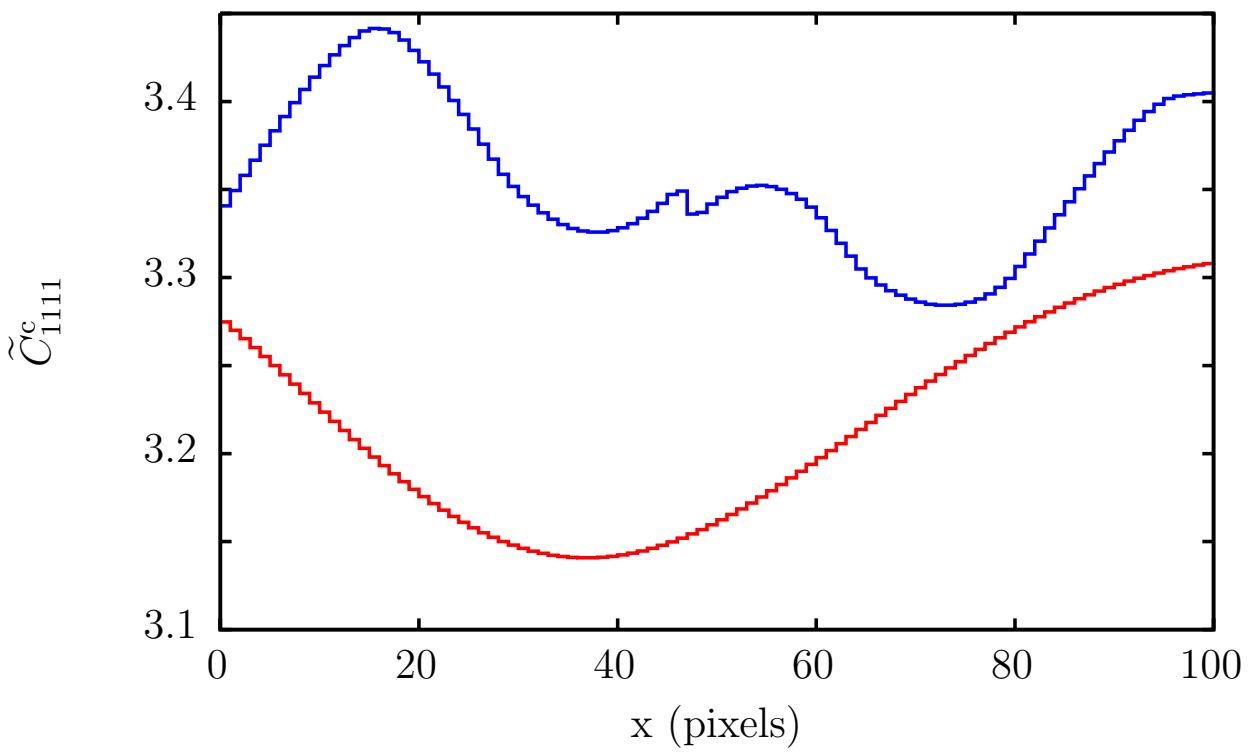

smooth gaussian filter

square window filter

(a) filter type effect: regularity

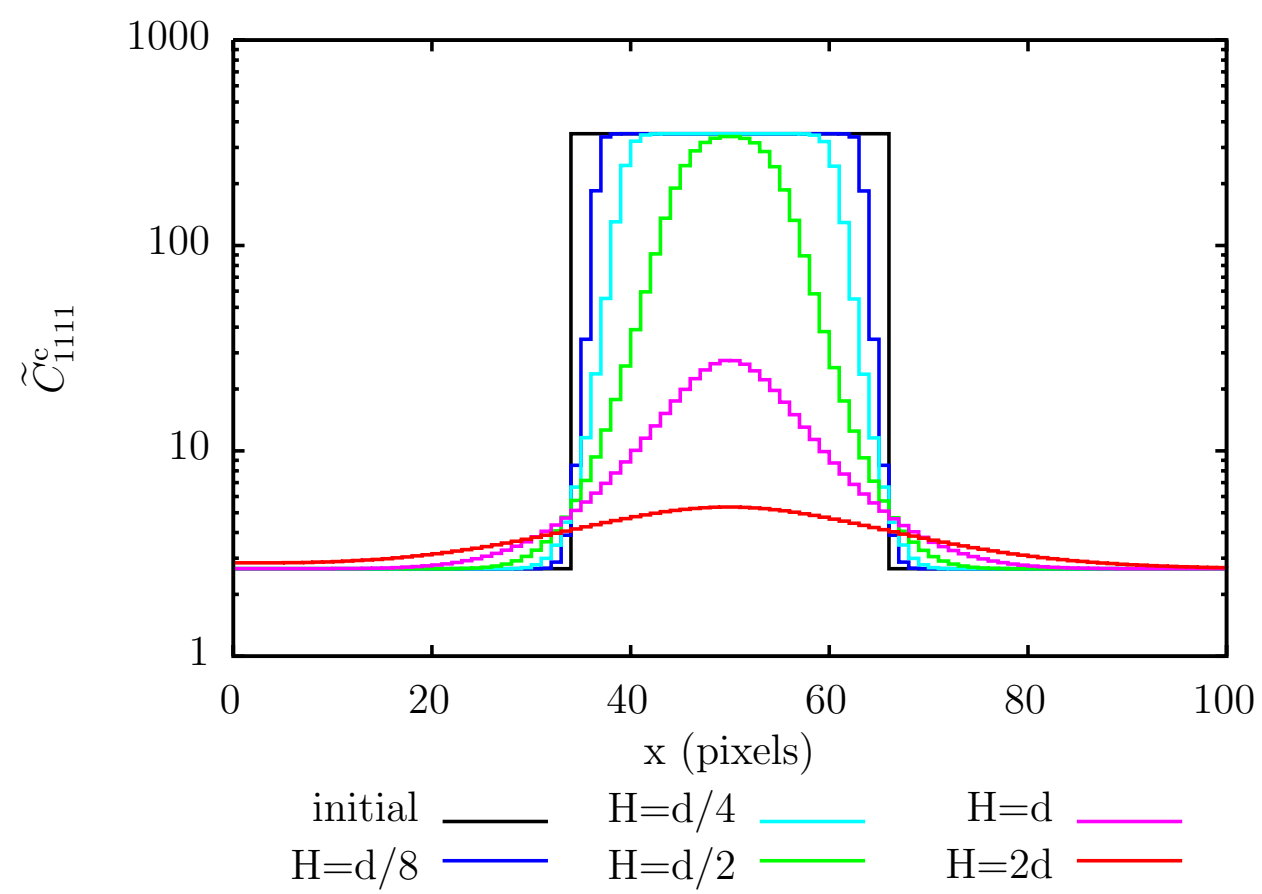

(b) filter size effect: smoothing

Figure 4: Cut of fields in Fig. 3 along a horizontal line. The diagrams (a) and (b) do not correspond to the same location. (a) Effect of filter type regularity on consistent filtered stiffness for a filter size $\mathrm{H}=128$ pixels, which is 4 times the fiber diameter $d$. (b) Effect of the filter size $H$ on the consistent filtered stiffness across a fiber for the smooth gaussian filter, expressed as a function of the fiber diameter $d$. 


\begin{tabular}{|c|c|c|c|c|c|c|c|c|c|c|c|c|}
\hline \multicolumn{3}{|c|}{$\widetilde{\mathbb{C}}^{\mathrm{k}}$} & \multicolumn{3}{|c|}{$\widetilde{\mathbb{C}}^{\mathrm{S}}$} & \multicolumn{3}{|c|}{$\widetilde{\mathbb{C}}^{\mathrm{C}}$} & \multicolumn{3}{|c|}{$\widetilde{\mathbb{C}}_{\text {sym }}^{\mathrm{C}}$} & \multirow[b]{2}{*}{$\Delta$ sym } \\
\hline$\|\Delta \mathbb{C}\|$ & $\Delta \kappa$ & $\Delta \mu$ & $\|\Delta \mathbb{C}\|$ & $\Delta \kappa$ & $\Delta \mu$ & $\|\Delta \mathbb{C}\|$ & $\Delta \kappa$ & $\Delta \mu$ & $\|\Delta \mathbb{C}\|$ & $\Delta \kappa$ & $\Delta \mu$ & \\
\hline $4.2 \mathrm{e}-12$ & $4.6 \mathrm{e}-13$ & $5.0 \mathrm{e}-12$ & $1.6 \mathrm{e}-12$ & $1.8 \mathrm{e}-13$ & $1.8 \mathrm{e}-12$ & 3.7e-12 & 3.1e-13 & $2.2 \mathrm{e}-12$ & $1.6 \mathrm{e}-11$ & $-9.2 \mathrm{e}-14$ & $2.5 \mathrm{e}-12$ & $1.5 \mathrm{e}-11$ \\
\hline $1.2 \mathrm{e}-5$ & $-1.2 \mathrm{e}-5$ & $-1.2 \mathrm{e}-5$ & $5.4 \mathrm{e}-6$ & $4.3 \mathrm{e}-6$ & $4.7 \mathrm{e}-6$ & $4.5 \mathrm{e}-10$ & $-4.7 \mathrm{e}-10$ & $1.9 \mathrm{e}-10$ & $1.4 \mathrm{e}-7$ & $-1.5 \mathrm{e}-7$ & $-1.1 \mathrm{e}-7$ & $3.8 \mathrm{e}-4$ \\
\hline $7.8 \mathrm{e}-5$ & $-7.9 \mathrm{e}-5$ & $-7.2 \mathrm{e}-5$ & $2.9 \mathrm{e}-5$ & $2.8 \mathrm{e}-5$ & $2.4 \mathrm{e}-5$ & $1.5 \mathrm{e}-9$ & $1.2 \mathrm{e}-9$ & $-4.6 \mathrm{e}-10$ & $8.7 \mathrm{e}-7$ & $-8.5-7$ & $-8.0 \mathrm{e}-7$ & $9.2 \mathrm{e}-4$ \\
\hline $4.6 \mathrm{e}-4$ & $-4.6 \mathrm{e}-4$ & $-4.5 \mathrm{e}-4$ & $1.5 \mathrm{e}-4$ & $1.5 \mathrm{e}-4$ & $1.4 \mathrm{e}-4$ & $1.4 \mathrm{e}-9$ & 1.0e-9 & $-2.0 \mathrm{e}-10$ & $5.1 \mathrm{e}-6$ & $-5.3 \mathrm{e}-6$ & $-4.5 \mathrm{e}-6$ & $2.0 \mathrm{e}-3$ \\
\hline $1.3 \mathrm{e}-3$ & $-1.1 \mathrm{e}-3$ & $-1.6 \mathrm{e}-3$ & $4.3 \mathrm{e}-4$ & $4.8 \mathrm{e}-4$ & $3.5 \mathrm{e}-4$ & $1.9 \mathrm{e}-8$ & $-1.2 \mathrm{e}-8$ & $1.2 \mathrm{e}-8$ & $2.1 \mathrm{e}-5$ & $-2.1 \mathrm{e}-5$ & $-2.0 \mathrm{e}-5$ & $3.5 \mathrm{e}-3$ \\
\hline $1.2 \mathrm{e}-3$ & $-8.9 \mathrm{e}-4$ & $-1.4 \mathrm{e}-3$ & $1.4 \mathrm{e}-3$ & $1.7 \mathrm{e}-3$ & $6.3 \mathrm{e}-4$ & $7.4 \mathrm{e}-6$ & $9.6 \mathrm{e}-7$ & $-9.3 \mathrm{e}-6$ & $3.7 \mathrm{e}-5$ & $-2.3 \mathrm{e}-5$ & $-5.0 \mathrm{e}-5$ & $5.3 \mathrm{e}-3$ \\
\hline $5.2 \mathrm{e}-4$ & $-3.6 \mathrm{e}-4$ & $-6.9 \mathrm{e}-4$ & $1.5 \mathrm{e}-3$ & $1.9 \mathrm{e}-3$ & $5.5 \mathrm{e}-4$ & $4.4 \mathrm{e}-6$ & $-1.1 \mathrm{e}-7$ & $-4.4 \mathrm{e}-6$ & $3.5 e-5$ & $-1.9 \mathrm{e}-5$ & $-4.9 \mathrm{e}-5$ & $4.3 \mathrm{e}-3$ \\
\hline $3.0 \mathrm{e}-4$ & $-2.3 \mathrm{e}-4$ & $-3.6 \mathrm{e}-4$ & $9.1 \mathrm{e}-4$ & $1.1 \mathrm{e}-3$ & $3.0 \mathrm{e}-4$ & $6.3 \mathrm{e}-6$ & $-5.5 \mathrm{e}-6$ & $-1.9 \mathrm{e}-6$ & $2.9 \mathrm{e}-5$ & $-2.0 \mathrm{e}-5$ & $-3.8 \mathrm{e}-5$ & $2.5 \mathrm{e}-3$ \\
\hline $2.7 \mathrm{e}-4$ & $-2.6 \mathrm{e}-4$ & $-2.7 \mathrm{e}-4$ & $3.8 \mathrm{e}-4$ & $4.7 \mathrm{e}-4$ & $1.8 \mathrm{e}-4$ & $1.9 \mathrm{e}-6$ & $-1.5 \mathrm{e}-6$ & $-1.1 \mathrm{e}-6$ & $2.2 \mathrm{e}-5$ & $-1.6 \mathrm{e}-5$ & $-2.8 \mathrm{e}-5$ & $1.0 \mathrm{e}-3$ \\
\hline
\end{tabular}

Table 1: Comparison of the initial problem homogenized tensor $\mathbb{C}^{\text {hom }}$ with the filtered problems homogenized tensor $\mathbb{C}^{\mathrm{c}, \text { hom }}$, $\mathbb{C}^{\mathrm{k}, \mathrm{hom}}, \mathbb{C}^{\mathrm{s}, \mathrm{hom}}$ and $\mathbb{C}_{\mathrm{sym}}^{\mathrm{c}, \mathrm{hom}}$. The isotropic bulk and shear homogenized modulii are also compared to check the validity of bounds. Concise notations refer to: $\|\Delta \mathbb{C}\|=\left\|\widetilde{\mathbb{C}}^{\text {type,hom }}-\mathbb{C}^{\text {hom }}\right\| /\left\|\mathbb{C}^{\text {hom }}\right\|, \Delta \kappa=\left(\tilde{\kappa}^{\text {type,hom }}-\kappa^{\text {hom }}\right) / \kappa^{\text {hom }}, \Delta \mu=\left(\tilde{\mu}^{\text {type, hom }}-\right.$ $\left.\mu^{\text {hom }}\right) / \mu^{\text {hom }}$ where type is either kinematic $(\mathrm{k})$, static $(\mathrm{s})$, consistent $(\mathrm{c})$ or consistent symmetrized $\left(\mathrm{c}_{\mathrm{sym}}\right)$ and $\Delta$ sym $=$ $\overline{\left\|\widetilde{\mathbb{C}}_{\mathrm{sym}}^{\mathrm{c}}-\mathbb{C}^{\mathrm{c}}\right\|} / \overline{\left\|\mathbb{C}^{\mathrm{c}}\right\|}$. The notation $\|\bullet\|$ refers to the $L^{2}$ norm.

\subsection{Homogenization of the filtered stiffness fields}

The filtered stiffness field obtained in section 4.2 are now input in our numerical solver to check the agreement with our theoretical analysis. The homogenized stiffness tensor $\mathbb{C}^{\text {hom }}$ obtained by the numerical homogenization of the initial problem is taken as a reference. When homogenizing the consistent filtered stiffness field, the homogenized stiffness of the initial problem is expected to be retrieved whatever the filter shape or size. In this section, dedicated to the verification of the consistency of numerical results with the filtering concepts, computations are carried out using the filtered stiffness fields obtained from the microstructure with cylindrical inclusions embedded in a matrix considered in section 4.2. The same discretization grids as for the initial homogenization problem resolution are used, so that of course no computational gain is expected at this point.

Four different filtered stiffness fields are tested: the three previously introduced consistent $\widetilde{\mathbb{C}}^{c}$, kinematic $\widetilde{\mathbb{C}}^{\mathrm{k}}$ and static $\widetilde{\mathbb{C}}^{\mathrm{s}}$ filtered stiffness fields. Additionally, the stiffness field $\widetilde{\mathbb{C}}_{\text {sym }}^{\mathrm{c}}$ obtained by taking the symmetric part of $\widetilde{\mathbb{C}}^{c}$ is tested to study the importance of the anti-symmetric part of $\widetilde{\mathbb{C}}^{c}$. The corresponding homogenized stiffness tensors are respectively denoted by $\widetilde{\mathbb{C}}^{\text {chhom }}, \widetilde{\mathbb{C}}^{\mathrm{k}, \text { hom }}, \widetilde{\mathbb{C}}^{\mathrm{s}, \text { hom }}$ and $\dot{\widetilde{\mathbb{C}}}_{\text {sym }}^{\text {chom }}$.

For each filtered stiffness field type $\mathrm{c}, \mathrm{k}, \mathrm{s}, \mathrm{c}_{\mathrm{sym}}$, three error measurements are monitored. First, the relative error between $\widetilde{\mathbb{C}}^{\text {type,hom }}$ and $\widetilde{\mathbb{C}}^{\text {hom }}$ defined as $\left\|\widetilde{\mathbb{C}}^{\text {type,hom }}-\mathbb{C}^{\text {hom }}\right\| /\left\|\mathbb{C}^{\text {hom }}\right\|$ using the $L^{2}$ norm. Then, the homogenized stiffnesses have been projected on the basis of isotropic $4^{\text {th }}$ order tensors in two dimensions to evaluate the homogenized bulk $(\kappa)$ and shear $(\mu)$ modulii. The signed relative errors $\left(\tilde{\kappa}^{\text {type,hom }-}\right.$ $\left.\kappa^{\text {hom }}\right) /\left(\kappa^{\text {hom }}\right)$ and $\left(\tilde{\mu}^{\text {type,hom }}-\mu^{\text {hom }}\right) /\left(\mu^{\text {hom }}\right)$ are monitored to check whether the bounds (22) are violated due to discretization. Finally, an additional indicator $\Delta$ sym is defined to study the non symmetry of the field $\widetilde{\mathbb{C}}^{c}$ as

$$
\Delta \text { sym }=\frac{\overline{\left\|\widetilde{\mathbb{C}}_{\mathrm{sym}}^{\mathrm{c}}-\mathbb{C}^{\mathrm{c}}\right\|}}{\overline{\left\|\mathbb{C}^{\mathrm{c}}\right\|}} .
$$

Results are presented in table 1 for exactly the same microstructure as previously studied, with a fiber to matrix stiffness contrast of 100 . The grid size is $512^{2}$ pixels, the fiber diameter is 32 pixels. Filter sizes $H$ from 2 to 512 pixels have been tested for the square window filter. Unpresented results for the smooth gaussian filter are very similar. While increasing the filter size $H$, the consistent filtered stiffness field only suffers from minor numerical errors which decrease with contrast. On the other hand, homogenization of the $\widetilde{\mathbb{C}}^{\mathrm{k}}$ and $\widetilde{\mathbb{C}}^{\mathrm{s}}$ fields induces errors several orders of magnitude higher which may not be attributed to numerical effects but bear the signature they are indeed not consistent. As seen from their sign, the isotropized modulii 


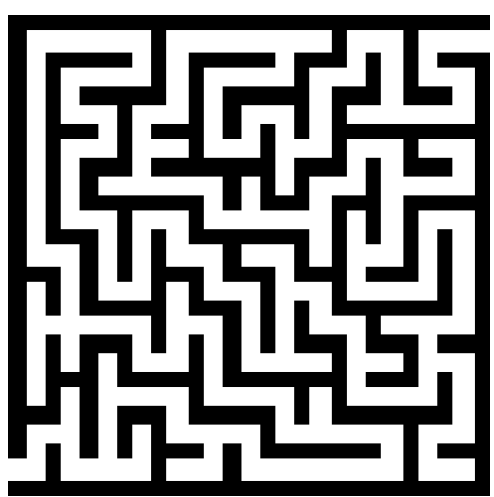

(a) $\mathbb{C}$

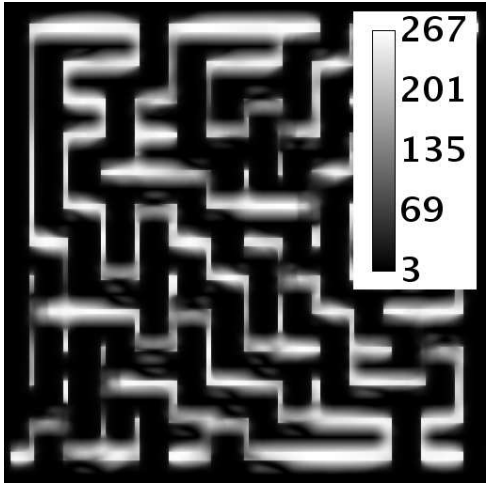

(b) $\widetilde{\mathbb{C}}^{\mathrm{s}}$

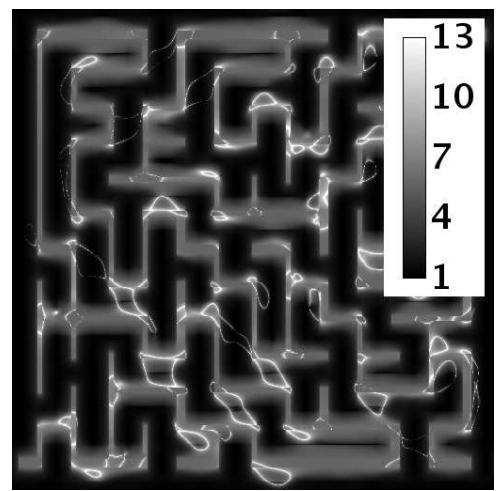

(c) $\log \left(\widetilde{\mathbb{C}}^{\mathrm{k}}\right)$

Figure 5: Example of complex microstructure. Component $C_{1111}$ of the initial and filtered stiffness fields with a square filter of size 16 pixels (roughly equal to beams width) in a $512^{2}$ discretization grid. (a) initial: the white phase has stiffness component $C_{1111}=267$ and the black phase $C_{1111}=2.67$. (b) static filter: smoothed stiffness field. (c) kinematic filter: strong oscillations (in gray level log scale!).

provide indeed the expected bounds (22), which are very sharp in this example. Incidentally, the error is maximum for filter sizes close to the diameter or radius of the fibers, which could be thought as some resonance effect $[4,7]$. Similar results are displayed in Fig. $7(\mathrm{a})$ and 7 (c) which were carried out over 50 realizations of 8-pixel diameter fibers with concentration $20 \%$ in a matrix, on $256^{2}$ discretization grids for various stiffness contrasts.

In the example with cylindrical inclusions embedded in matrix, where a low concentration of inclusion has been considered, the non symmetry indicator (23) remains low. However, the non symmetry of $\widetilde{\mathbb{C}}^{c}$ is pronounced close to the matrix-fiber boundaries, where the field fluctuations are the highest. The above example thus suffers from averaging effects. In turn, more sophisticated structures such as complex intrication of the two phases (Fig. 5) have been put at test. The non symmetry effect was far more pronounced, especially for filter sizes close to microstructure characteristic size, where the non symmetry indicator (23) exceeded 0.15. In the mean time, the error in the homogenized stiffness using the $\widetilde{\mathbb{C}}^{\mathrm{k}}$, $\widetilde{\mathbb{C}}^{\mathrm{k}}$ and $\widetilde{\mathbb{C}}_{\text {sym }}^{\mathrm{c}}$ was two to three orders of magnitude higher than in the previous example. Additionally, field singularities and sharp fluctuations could not be captured finely enough at contrast 100 between the two phases with a $512^{2}$ discretization grid. These additional numerical experiments raise open issues about the numerical evaluation of the filtered stiffnesses from discretized fields.

\subsection{Sampling}

The smoothing and contrast reducing effect of filtering observed in Figs. 3 and 4 suggests a coarser discretization of the filtered field for the subsequent computation of the homogenized stiffness.

For sufficiently large windows, the consistent filtered problem $\left(\tilde{P}^{\mathrm{c}}\right)$, whose homogenization yields the exact homogenized stiffness, could be approximated by a so-called sampled filtered problem. The sampled filtered problem is defined by the extraction of the consistent filtered stiffness at sampling points regularly positioned on a coarse discretization grid (see Fig. 6). Just like in two step numerical homogenization methods, the filtered stiffness $\widetilde{\mathbb{C}}^{\mathrm{c}}\left(\boldsymbol{z}_{s}\right)$ of each sampling point $\boldsymbol{z}_{s}$ is assigned to a corresponding coarse element. Similar procedures with the kinematic or static filtered stiffnesses can also be devised. Sampling size is defined as the size of the elements of the coarse discretization grid. Strictly speaking, sampling will cause the loss of the consistency or bound status of the filtered stiffness, for some information is lost. However, reasonable combination of filter and sampling size should provide fair estimates of the homogenized stiffness.

Results in Fig. 7 show the relative error obtained with and without sampling. The sampling size is taken as the filter size, which is the coarsest grid size which could be reasonably used. Other sampling sizes (allowing for the overlap of the domains on which the filters act) could however be used, depending on the target final error on the homogenized elastic properties. The effect of contrast and filter size to fiber 

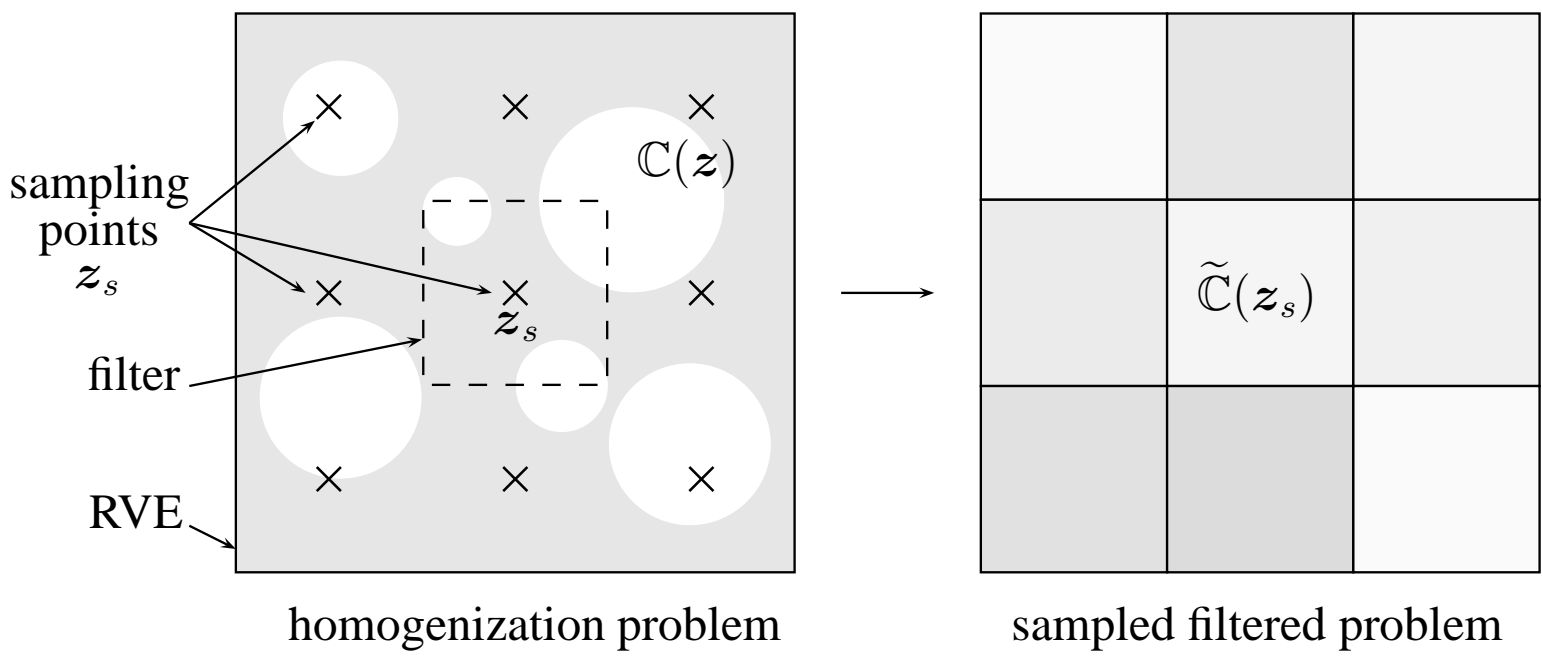

Figure 6: Sampling strategy: sampling points regularly spaced in the RVE at a coarse discretization distance and filtering at the sampling points only.

diameter $H / d$ is studied. The cell resonance effect in two step numerical homogenization methods is also observed here. For intermediate sampling size, not shown here, the error is between these two cases. The consistent status of the homogenized stiffness is lost with sampling, and $\widetilde{\mathbb{C}}^{\mathrm{c}}, \widetilde{\mathbb{C}}^{\mathrm{k}}$ or $\widetilde{\mathbb{C}}^{\mathrm{s}}$ lead to similar errors, although $\widetilde{\mathbb{C}}^{\mathrm{c}}$ seems to perform slightly better at low contrast. Then, ability to manage high contrast effects takes over and $\widetilde{\mathbb{C}}^{\mathrm{k}}$ or $\widetilde{\mathbb{C}}^{\mathrm{s}}$ should be used accordingly.

The effect of the filter regularity and anisotropy on estimations of the homogenized stiffness from sampled filtered stiffness fields has not been assessed, but is thought to be an interesting prospect.

Note that translating the coarse sampling grid would result in a different "discretization" of the filtered stiffness field and thus to a different estimate of $\mathbb{C}^{\text {hom }}$. We believe that the order of magnitude of the error on $\mathbb{C}^{\text {hom }}$ is the same in both cases. The average of estimates of $\mathbb{C}^{\text {hom }}$ for many translations of the sampling grid might reduce the error, but at an increased computational cost and without guarantee of a sharp reduction of the error. Instead, we believe this computational effort would be better spent on a refinement the sampling grid for a fixed filter size. Indeed, it would provide a better "discretization" of the filtered stiffness field and likely a better estimate of $\mathbb{C}^{\text {hom }}$.

It should be noted that, disregarding boundary condition issues, the two step numerical homogenization methods presented in Refs. [4-13] could be seen as sampling the filtered problem with a square window filter. Provided the filtered stiffness is closely related to the coarse element effective stiffness, the filtering point of view provides another framework for the study of two step numerical homogenization methods. In the next section, cost efficient estimates of $\widetilde{\mathbb{C}}\left(\boldsymbol{z}_{s}\right)$ are proposed.

\section{Efficient evaluation of the filtered stiffness}

In the present section, for any sampling point $\boldsymbol{z}_{s}$, we propose to estimate $\widetilde{\mathbb{C}}\left(\boldsymbol{z}_{s}\right)$ from the solution to a local problem of elastic equilibrium. This local problem is formulated on a small domain surrounding the sampling point $\boldsymbol{z}_{s}$. It involves a domain much smaller than the RVE, thus reducing significantly the cost of evaluating $\widetilde{\mathbb{C}}\left(\boldsymbol{z}_{s}\right)$. The resulting estimate of $\widetilde{\mathbb{C}}\left(\boldsymbol{z}_{s}\right)$, which will be denoted by $\widehat{\mathbb{C}}\left(\boldsymbol{z}_{s}\right)$, can be seen as the effective stiffness used in two step numerical homogenization methods [6-13].

\subsection{The local problem and oversampling}

A local problem is classically introduced in two step numerical homogenization methods to capture the fine scale field fluctuations. Its solution is used either to produce FEM shape functions for the coarse discretization $[4,5]$ or to compute effective stiffness for each coarse element [6-13]. 


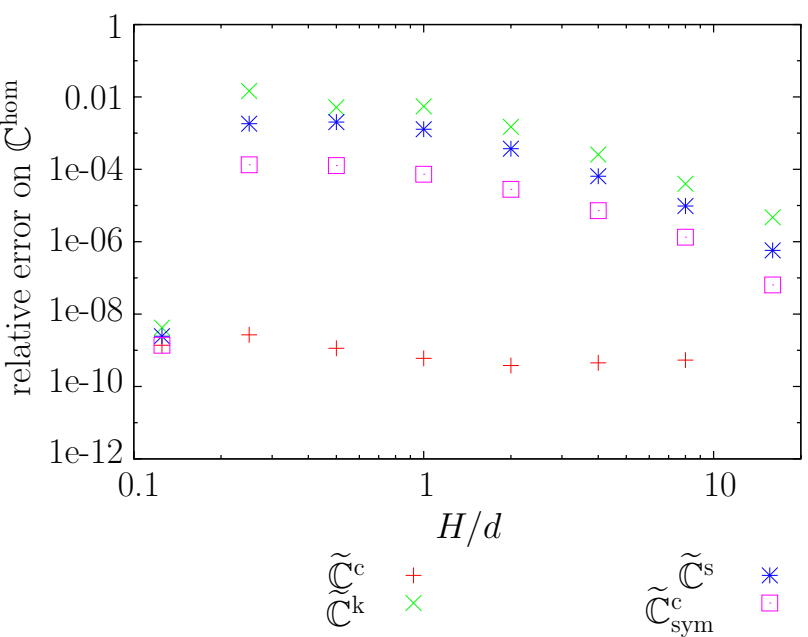

(a) contrast 0.1

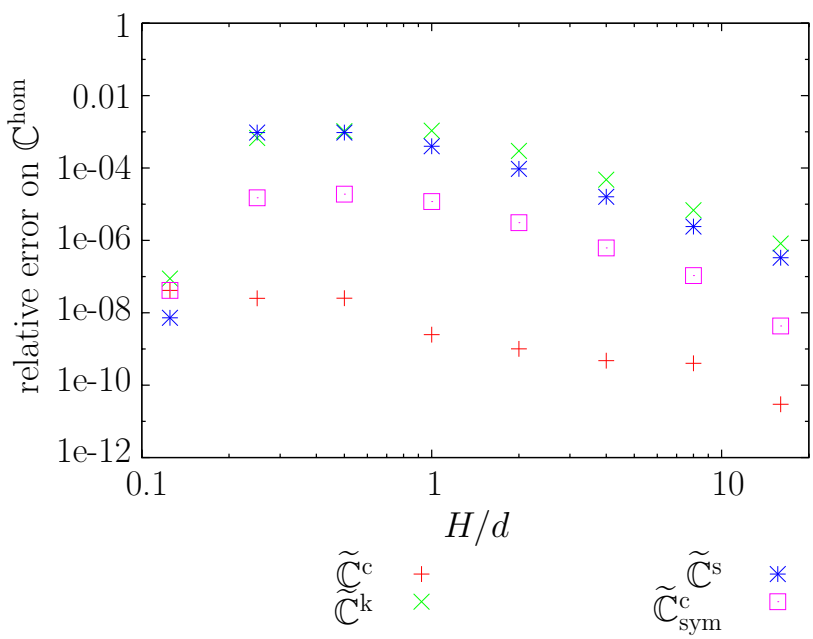

(c) contrast 10

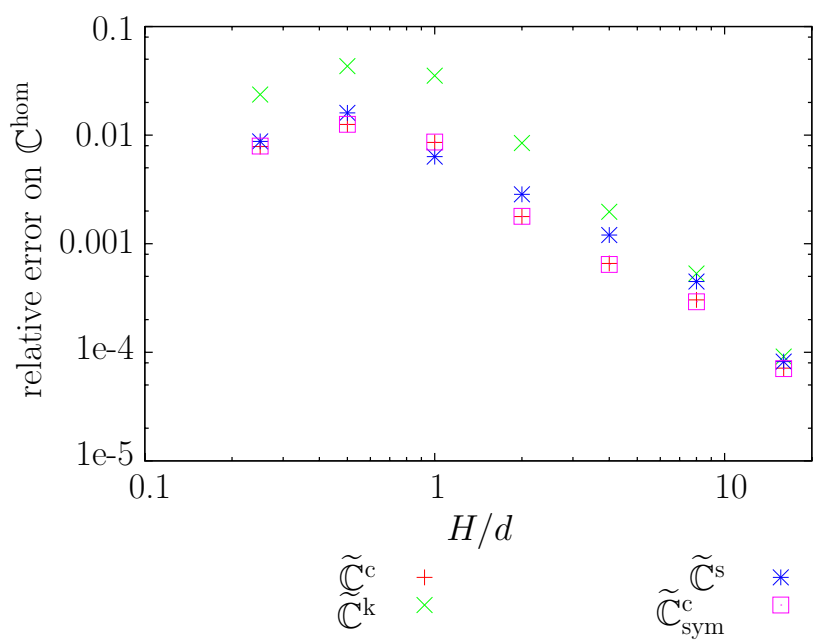

(b) contrast 0.1 sampled

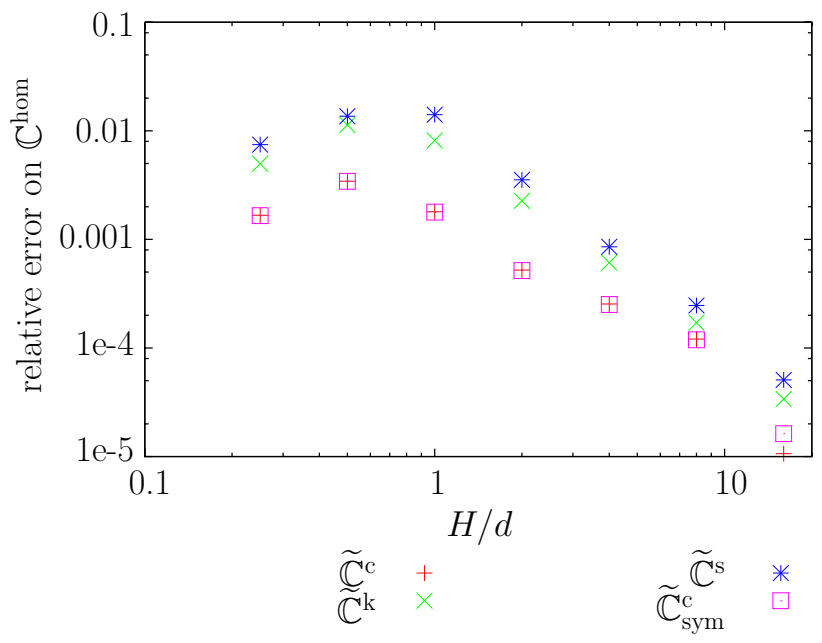

(d) contrast 10 sampled

Figure 7: Relative error on the homogenized stiffness $\mathbb{C}^{\text {hom }}$ resulting from the homogenization of the filtered stiffness fields. The contrast refers to the ratio of the fibers over matrix young modulii, for a Poisson ratio of 0.2. Sampling size is equal to filter size in the second column, while no sampling is performed in the first column. Mean of the error over 50 realizations of 8 pixels diameter cylindrical fibers embedded in a homogeneous matrix on a $256^{2}$ pixels grid, with fiber concentration of $20 \%$. 
In the literature, several types of local problems have been introduced. Their main differences are the type of boundary conditions applied, the presence or absence of oversampling and filtering procedures and the definition of the coarse element effective stiffness.

Three definitions of the coarse element effective stiffness $\widehat{\mathbb{C}}$ are possible:

$$
\begin{array}{lll}
\text { direct } & \bar{\sigma} & =\widehat{\mathbb{C}}: \bar{\epsilon} \\
\text { kinematic } & \bar{\epsilon}: \widehat{\mathbb{C}}: \bar{\epsilon} & =\overline{\epsilon: \mathbb{C}: \epsilon} \\
\text { static } & \bar{\sigma}: \widehat{\mathbb{C}}^{-1}: \bar{\sigma} & =\overline{\sigma: \mathbb{C}^{-1}: \sigma}
\end{array}
$$

where $\sigma$ and $\epsilon$ are the stress and strain solutions to the local problem and volume averages are performed on the coarse element.

Uniform strain (KUBC) or stress (SUBC) boundary conditions applied directly at the boundary of the coarse element have been shown to introduce a systematic bias (i.e. overestimation or underestimation) $[10,19,20]$. Namely, the use of KUBC (resp. SUBC) together with a kinematic (resp. static) definition of the effective stiffness and subsequent homogenization leads to upper (resp. lower) bounds on the homogenized stiffness $[19,20]$.

Periodic boundary conditions appear to be free of systematic bias [10] and have been widely used in such context [1, 6-8, 10-13]. Still, for all types of boundary conditions, undesirable effects appear within a boundary layer.

To partially circumvent undesirable boundary conditions effect, $[4,7,8]$ resorted to the oversampling strategy, while $[1,10,14,15,21]$ truncated (or eroded) the averages of the solutions to the local problems. More generally, [10] proposed to filter the averages in the left hand sides of (24).

The application of periodic boundary conditions directly at the boundary of the coarse element results in an equivalence between the direct, kinematic and static definitions of $\widehat{\mathbb{C}}$. However, this no longer holds whenever oversampling or filtering is performed [14]. In this case, the direct definition of $\widehat{\mathbb{C}}$ is no longer ensured to be definite positive.

While resorting to a two-step homogenization method based on coarse elements effective properties $\widehat{\mathbb{C}}$, convergence rates of the error on the homogenized properties have been shown [7] to be

$$
\left\|\widehat{\mathbb{C}}^{\text {hom }}-\mathbb{C}^{\text {hom }}\right\|=C_{1} \epsilon / H+C_{2} H+C_{3},
$$

where $\epsilon$ is the size of the fluctuations of the stiffness field and $H$ the size of the coarse element. This result applies both in the presence or absence of oversampling. The term $C_{1} \epsilon / H$ in (25) is called the cell-resonance effect. The two other terms are due to the non-conformance of the boundary conditions applied to the local problem. Oversampling reduces the prefactor $C_{1}[7,10]$, which is otherwise dominant in the absence of oversampling.

\subsection{Cost efficient estimates of the filterered stiffness}

Drawing from two-step homogenization methods, we define a local problem for each sampling point $\boldsymbol{z}_{s}$ to derive cost efficient estimates $\widehat{\mathbb{C}}\left(\boldsymbol{z}_{s}\right)$ of the filtered stiffness $\widetilde{\mathbb{C}}\left(\boldsymbol{z}_{s}\right)$. The problem is defined on a small domain $S$ surrounding the sampling point $\boldsymbol{z}_{s}$. The filters under consideration are supported in domains of finite extent. In the line of reasoning of some two step numerical homogenization methods [4, 5, 7, 8, 10], we additionally propose to resort to a strategy inspired from oversampling methods. Namely, the domain $S$ will be made of the domain on which the filter centered in $\boldsymbol{z}_{s}$ acts, augmented by an oversampling region of thickness $t$ (Fig. 8). Oversampling is crucial to account for the intrinsically non-local definition of the filtered stiffness and to reduce boundary effects.

The choice of boundary conditions is then critical to the definition of the local problem. In the remainder of this paper, periodic boundary conditions are applied on the boundary of the oversampled domain $S(t)$. Indeed, previous studies [10] show that these boundary conditions tend to minimize boundary effects.

Based on our filtering analysis, we propose three possible definitions of the estimate $\widehat{\mathbb{C}}$.

The first definition of $\widehat{\mathbb{C}}$ is based on direct filtering. The solution to the local problem is characterized by the strain localization tensor $\mathbb{A}(t)$ which obviously depends on $S(t)$. Following the analysis that lead to 


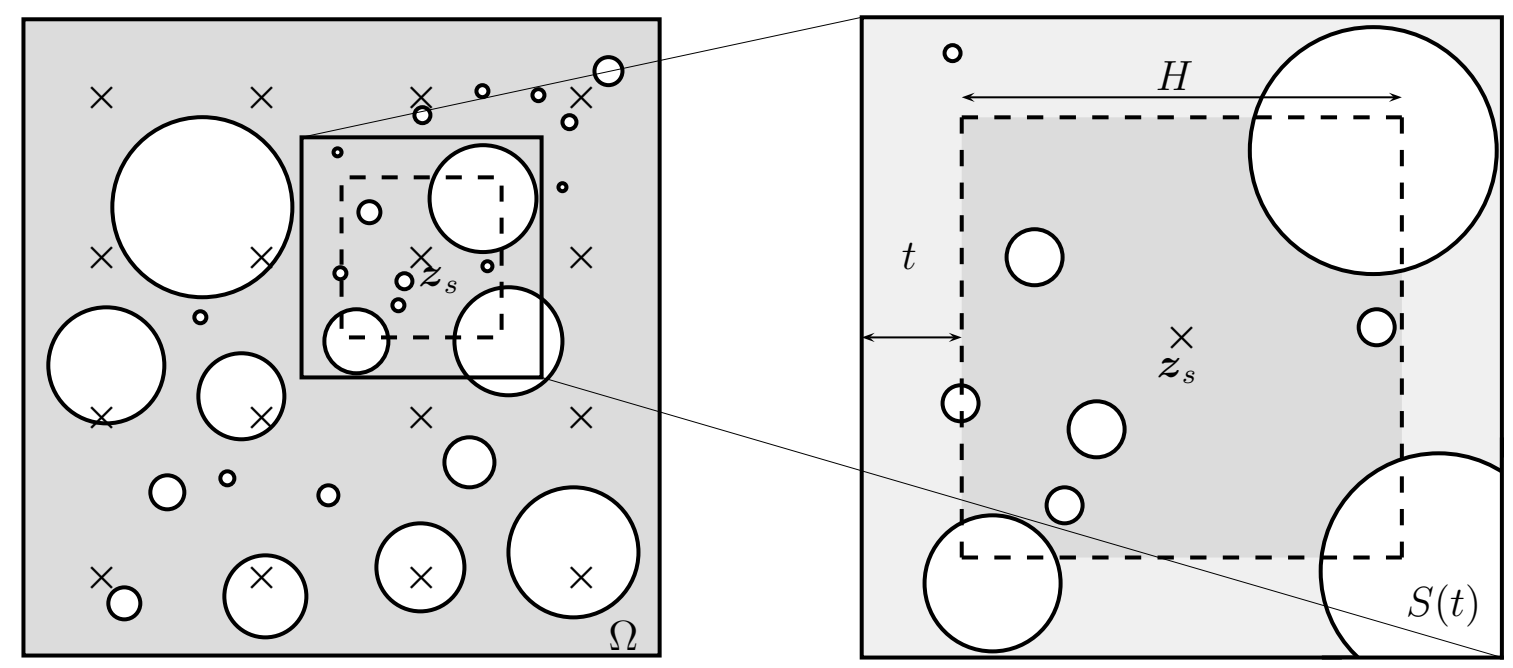

Figure 8: Estimation of the filtered stiffness at the sampling point $\boldsymbol{z}_{s}$ by resolution of a local problem. The dotted square represents the domain on which the filter centered in $\boldsymbol{z}_{s}$ acts. The small domain $S(t)$ surrounding the sampling point $\boldsymbol{z}_{s}$, on which the local problem is defined, is made of the domain on which the filter centered in $\boldsymbol{z}_{s}$ acts augmented by an oversampling region of thickness $t$ to reduce boundary effects.

the introduction of the consistent filtered stiffness, an estimate $\widehat{\mathbb{C}}^{c}\left(\boldsymbol{z}_{s}, t\right)$ of the consistent filtered stiffness $\widetilde{\mathbb{C}}^{\mathrm{C}}\left(\boldsymbol{z}_{s}\right)$ at the sampling point the point $\boldsymbol{z}_{s}$ is proposed as

$$
\widehat{\mathbb{C}}^{\mathrm{c}}\left(\boldsymbol{z}_{s}, t\right)=(\mathbb{C}: \mathbb{A}(t))^{*}\left(\boldsymbol{z}_{s}\right):\left(\mathbb{A}(t)^{*}\right)^{-1}\left(\boldsymbol{z}_{s}\right) .
$$

Practically, the estimate $\widehat{\mathbb{C}}^{c}\left(\boldsymbol{z}_{s}, t\right)$ is computed as follows:

1. solve local problems centered at the point $\boldsymbol{z}_{i}$ with boundary conditions corresponding to unit mesoscopic loads $\boldsymbol{E}=\boldsymbol{e}_{x} \otimes \boldsymbol{e}_{x}, \boldsymbol{e}_{y} \otimes \boldsymbol{e}_{y}, \ldots$

2. filter the solution stress field $\boldsymbol{\sigma}^{*}\left(\boldsymbol{z}_{i}\right)=(\rho * \boldsymbol{\sigma})\left(\boldsymbol{z}_{i}\right)$.

3. filter the solution strain field $\boldsymbol{\epsilon}^{*}\left(\boldsymbol{z}_{i}\right)=(\rho * \boldsymbol{\epsilon})\left(\boldsymbol{z}_{i}\right)$.

4. define the mesoscopic effective stiffness estimate $\widehat{\mathbb{C}}^{c}\left(\boldsymbol{z}_{i}\right)$ such that $\boldsymbol{\sigma}^{*}\left(\boldsymbol{z}_{i}\right)=\widehat{\mathbb{C}}^{c}\left(\boldsymbol{z}_{i}\right): \boldsymbol{\epsilon}^{*}\left(\boldsymbol{z}_{i}\right)$. Finally,

$$
\widehat{\mathbb{C}}^{c}\left(\boldsymbol{z}_{i}\right)=(\mathbb{C}: \mathbb{A})^{*}:\left(\mathbb{A}^{*}\right)^{-1} .
$$

This effective stiffness $\widehat{\mathbb{C}}^{c}\left(\boldsymbol{z}_{s}, t\right)$ differs from the effective stiffness $\mathbb{C}^{*}\left(\boldsymbol{z}_{i}\right)$ defined in previous filtering strategies $[1,10,21]$ which, using our formalism, amounts to:

1. solve local problems centered at the point $\boldsymbol{z}_{i}$ with boundary conditions corresponding to unit mesoscopic loads $\boldsymbol{E}=\boldsymbol{e}_{x} \otimes \boldsymbol{e}_{x}, \boldsymbol{e}_{y} \otimes \boldsymbol{e}_{y}, \ldots$

2. filter the solution stress field $\boldsymbol{\sigma}^{*}\left(\boldsymbol{z}_{i}\right)=(\rho * \boldsymbol{\sigma})\left(\boldsymbol{z}_{i}\right)$.

3. define the mesoscopic effective stiffness $\mathbb{C}^{*}\left(\boldsymbol{z}_{i}\right)$ such that $\boldsymbol{\sigma}^{*}\left(\boldsymbol{z}_{i}\right)=\mathbb{C}^{*}\left(\boldsymbol{z}_{i}\right): \boldsymbol{E}$. Finally,

$$
\mathbb{C}^{*}\left(\boldsymbol{z}_{i}\right)=(\mathbb{C}: \mathbb{A})^{*}
$$

The two stiffnesses $(27)$ and $(28)$ differ by the term $\left(\mathbb{A}^{*}\right)^{-1}$. The term $\left(\mathbb{A}^{*}\right)^{-1}$ is deemed crucial for the types of microstructures with high stiffness contrast considered in this article.

Whenever $S(t)$ is the entire RVE $\Omega$, the estimate $\widehat{\mathbb{C}}^{\mathrm{c}}\left(\boldsymbol{z}_{s}, t\right)$ is by definition equal to the consistent filtered stiffness $\widetilde{\mathbb{C}}^{\mathrm{c}}\left(\boldsymbol{z}_{s}\right)$. In general, however, the sampled field $\widehat{\mathbb{C}}^{\mathrm{c}}(t)$ is not consistent in the sense that its homogenization will not yield the exact homogenized stiffness. As seen previously (Fig. 7 and Tab. 1), the 
anti-symmetric part plays an important role when the whole field of consistent filtered stiffness is used, but a minor one as soon as sampling of the field is made. Hence the symmetry enforcement required in Chen et al. [9] may be done, at least for the simple microstructures presented in this article.

The second definition of $\widehat{\mathbb{C}}$ is an estimate of the kinematic filtered stiffness $\widetilde{\mathbb{C}}^{\mathrm{k}}$ such

$$
\widehat{\mathbb{C}}^{\mathrm{k}}\left(\boldsymbol{z}_{s}, t\right)={ }^{t}\left(\mathbb{A}(t)^{*}\right)^{-1}\left(\boldsymbol{z}_{s}\right):\left({ }^{t} \mathbb{A}(t): \mathbb{C}: \mathbb{A}(t)\right)^{*}\left(\boldsymbol{z}_{s}\right):\left(\mathbb{A}(t)^{*}\right)^{-1}\left(\boldsymbol{z}_{s}\right),
$$

which is symmetric and positive definite by construction. Additionally, $\widehat{\mathbb{C}}^{\mathrm{k}}$ is suited for coarse elements which contain infinitely stiff phases.

The third definition is based on the stress localization tensor $\mathbb{B}(t)$ and is not indeterminate in the presence of pores

$$
\widehat{\mathbb{C}}^{\mathrm{s}}\left(\boldsymbol{z}_{s}, t\right)=\mathbb{B}(t)^{*}\left(\boldsymbol{z}_{s}\right):\left[\left({ }^{t} \mathbb{B}(t): \mathbb{S}: \mathbb{B}(t)\right)^{*}\left(\boldsymbol{z}_{s}\right)\right]^{-1}:{ }^{t} \mathbb{B}(t)^{*}\left(\boldsymbol{z}_{s}\right)
$$

These two last definitions are equivalent to $\widetilde{\mathbb{C}}^{\mathrm{k}}$ and $\widetilde{\mathbb{C}}^{\mathrm{s}}$ respectively when $S(t)=\Omega$. They provide good alternatives to study extreme cases encountered while upscaling (pores or infinitely stiff inclusions). This analysis complements the discussion in Chen et al. [9] whether using transmissibilities or permeabilities as effective properties of the coarse grid in two step darcean transport homogenization.

It should be noted that the filtered stiffness fields $\widetilde{\mathbb{C}}$ introduced in Sec. 3 are free of undesirable boundary condition effects, but are only a theoretical construction. On the other hand, the practical estimates $\widehat{\mathbb{C}}$ of the filtered stiffness $\widetilde{\mathbb{C}}$ proposed in this section do suffer from local problem boundary condition effects. The effect of boundary conditions will be numerically studied as a function of the oversampling thickness $t$ in the next section.

Namely, the convergence with respect to $t$ of the estimates $\widehat{\mathbb{C}}\left(\boldsymbol{z}_{s}, t\right)$ defined from the local problem to $\widetilde{\mathbb{C}}\left(\boldsymbol{z}_{s}\right)$ defined from the homogenization problem is expected and numerically studied in the following section.

\subsection{Local convergence with oversampling of the estimate stiffness to the filtered stiffness}

As expected, error on the estimation of $\widetilde{\mathbb{C}}$ by $\widehat{C}(t=0)$ without oversampling diminishes with filter size by dilution of the boundary effects (Fig. 9). Similar results had been noticed by Graham-Brady et al. [12] for coarse elements effective properties. For oversampling sizes $t$ from 0 to the particle diameter $d$, increasing $t$ allows to correct this filter size effect. As a comparison, [14] suggests using a thickness of 0.4 times the fiber diameter in a similar configuration. For $t$ larger than $d, \widehat{\mathbb{C}}(t)$ has an overall convergence towards $\widetilde{\mathbb{C}}$, although the error decrease is not monotonous. Filter size effect seems to play a minor role for large oversampling sizes. The effect of the filtered stiffness type, consistent, kinematic or static, is unclear and seems to depend on configurations.

\section{Application to a two step numerical homogenization method}

\subsection{The two step numerical homogenization method and its strategy}

The oversampling strategy studied in the previous section provides a way to estimate the filtered stiffness field at sampling points by solving decoupled smaller problems. The local problems, much smaller than the homogenization problem, can be solved with a fine discretization. The choice of the fine discretization influences the accuracy of the final estimation of the homogenized stiffness and obviously the computational cost of the method. A detailed discussion on convergence rates of the solution with respect to the fine discretization has been proposed by Abdulle [22] in the case of a separation of scales and can help to guide this choice. Then, as in grid-block [6-10] or moving window [11-13] methods, the sampled estimates of the filtered stiffness are affected to coarse elements corresponding to the sampling points. Resolution gives an estimate of the homogenized stiffness. We aim to provide guidelines for the three remaining parameters that have to be adjusted:

1. filter size, 


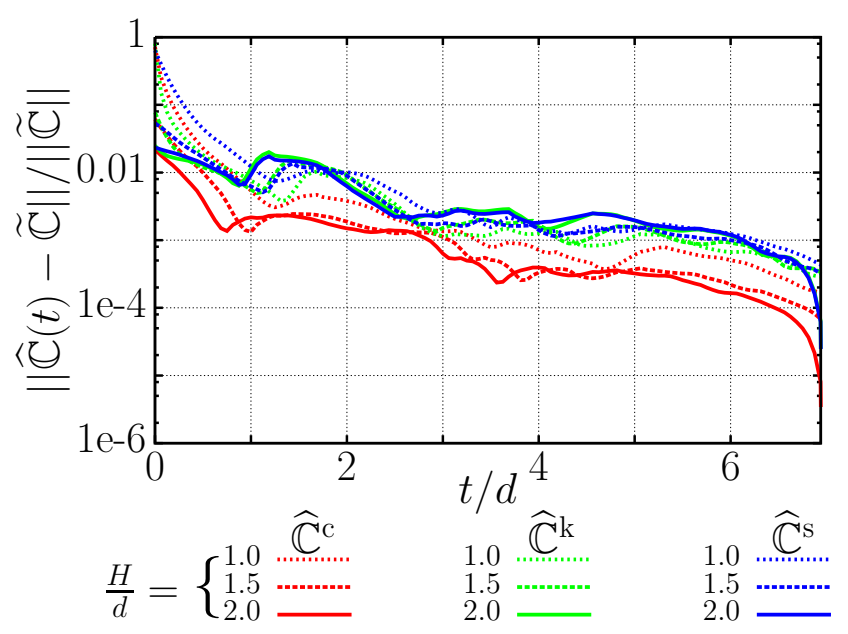

(a) isolated grain, contrast 100

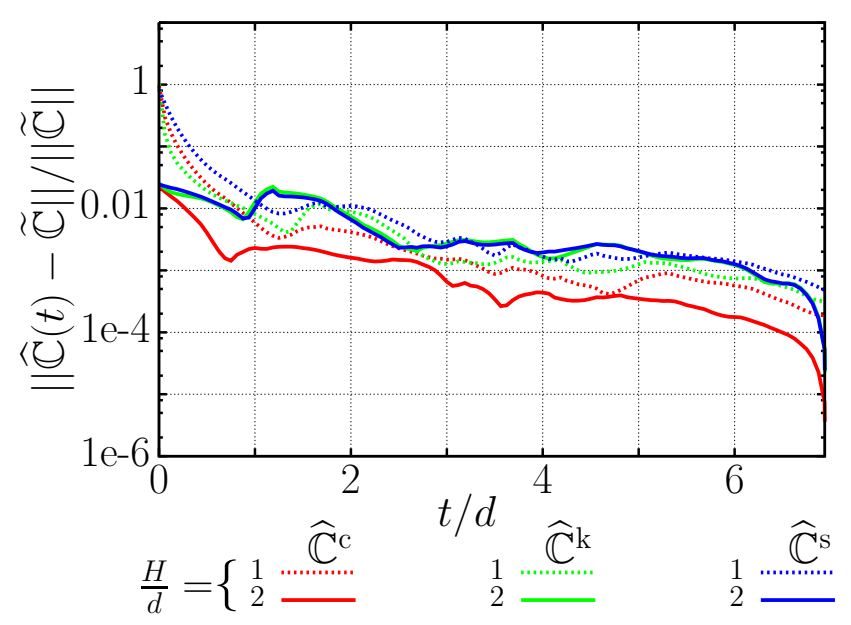

(c) isolated grain, contrast 10000

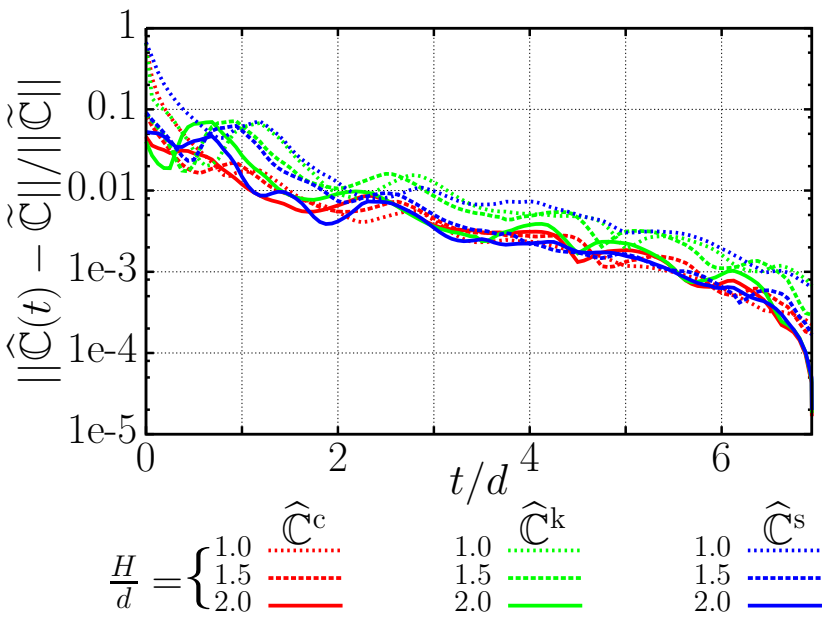

(b) surrounded grain, contrast 100

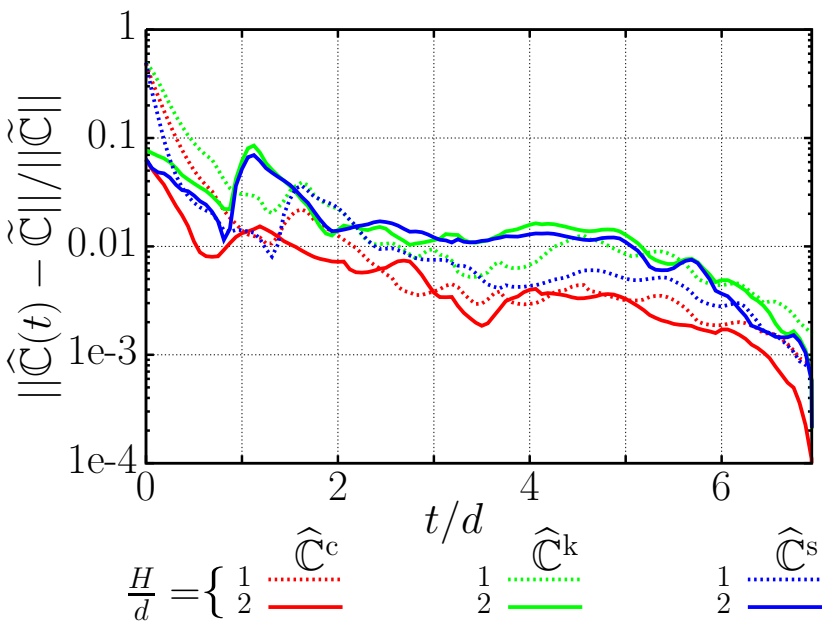

(d) isolated grain, contrast 0.01

Figure 9: Local convergence of the estimate stiffness obtained by oversampling to the filtered stiffness as a function of the oversampling border region size $t$ normalized by the fiber diameter $d$. The microstructure is made of cylindrical fibers of diameter $d=32$ pixels embedded in a homogeneous matrix. Fiber concentration is $25 \%$, the homogenization grid has $512^{2}$ pixels. Different square window sizes $H$ are tested and effect of contrast is studied. The sampling point is either centered on an isolated fiber far from other fibers or on a fiber closely surrounded by other fibers, showing the influence of the local configuration. 
2. coarse discretization size, or equivalently, distance between sampling points,

3. oversampling border region thickness.

The coarse discretization size should be chosen smaller than the filter size to keep the error reasonable (Fig. 7). However too small coarse grid size imply large overhead computations since the border region to coarse element size is increased, making the method inefficient. A good error/computational time trade off seems to choose the filter size equal to the coarse grid size. The error sources are then twofolds [7]

1. the cell resonance effect, which is governed by the ratio of the sampling/filter size to the length-scale of the microstructure fluctuations (see Fig. 7),

2. the non-conformation of the boundary conditions applied to the different local problems, which is governed by the ratio of the thickness of the oversampling region to the length-scale of the microstructure fluctuations (see Fig. 9).

Again an error/computational cost trade off appears. On one hand, the computational time and memory occupation of the proposed numerical method are known in advance by the user. On the other hand, the two sources of error are a priori unknown but can be estimated from guideline curves relevant to the studied microstructure, such as Fig. 7 and 9 for the example of composites made of inclusions embedded in a matrix.

Additionally, the choice of the effective stiffness between $\widehat{\mathbb{C}}^{c}(t), \widehat{\mathbb{C}}^{\mathrm{k}}(t)$ or $\widehat{\mathbb{C}}^{\mathrm{s}}(t)$ is to be driven for each coarse element by phase contrasts as exposed previously.

\subsection{Numerical results}

The two step numerical homogenization method is first tested on a composite made of fibers embedded in a homogeneous matrix with a volume fraction of fibers of $20 \%$. The fine discretization grid of the whole RVE is $256^{2}$ pixels and all fibers have a diameter of 8 pixels. Results in Fig. 10 presents the relative error on the homogenized stiffness while resorting to the two step method, for two severe inclusion to matrix stiffness contrast. A total of 20 random realizations have been studied. For each realization $\omega$, the norm of the relative error $\left\|\widehat{\mathbb{C}}^{\mathrm{c}, \operatorname{hom}}(\omega)-\mathbb{C}^{\operatorname{hom}}(\omega)\right\| /\left\|\mathbb{C}^{\text {hom }}(\omega)\right\|$ has been computed. Fig. 10 presents the statistical average of the norm of the relative error for the 20 realizations. The estimate $\widehat{\mathbb{C}}^{c}(t)$ of the consistent filtered stiffness is used for the coarse grid effective properties. A square window filter of size equal to the coarse discretization size $H$ is chosen and varied from 2 to 16 times the diameter of the inclusions. For each of these choices, oversampling region sizes $t$ from half to two diameters are tested and results are compared to those with the sampled filtered stiffness.

Results in Fig. 10 indicate that the sampled filtered stiffness obtained by a full direct computation always perform better than its estimates obtained by local problems with oversampling. The major reason is thought to rely in the absence of non-conforming boundary conditions effects in the sampled filtered stiffness. In other words, the term $C_{2} H+C_{3}$ in $(25)$ is absent for $\widetilde{\mathbb{C}}^{c}$ and the prefactor $C_{1}$ in the cellresonance effect is low. The slope of $\widetilde{\mathbb{C}}^{c}$ with respect to $H$ in log-log scale is -1 , which is in agreement with the $\epsilon / H$ convergence where the size of the heterogeneities $\epsilon$ is $d$. Clearly, the sampled filtered stiffness $\widetilde{\mathbb{C}}^{c}$ is not known in practice but the comparison to the results obtained with the estimate stiffness $\widehat{\mathbb{C}}^{c}$ is instructive. Indeed, for coarse discretization size $H$ close to the fiber diameter $d$ the slope in $\epsilon / H$ of the $\widehat{\mathbb{C}}^{\mathrm{c}}(t=0)$ curve indicates the cell-resonance effect drives the error when no oversampling is performed. Oversampling provides a good boundary condition error correction and is even an excellent alternative in terms of errors. While oversampling, the cell-resonance effect prefactor $C_{1}$ in (25) is reduced. As a result, as $t$ increases the cell-resonance effect is less and less dominant over the non-conformation of the boundary conditions term $\mathrm{C}_{2} \mathrm{H}+\mathrm{C}_{3}$, resulting in a decrease in the slope. However, overhead computations associated to the border region may cause computational time to be prohibitive, so larger coarse discretization size might be preferred in the limit of available memory.

The ability of the two step homogenization method to deal with microstructures having a wide range of heterogeneity sizes, as initially sought (see Fig. 1), is then tested. To this extent, a composite made of fibers embedded in a homogeneous matrix is considered, with two diameters of fibers. A ratio $d_{\max } / d_{\min }=8$ in diameters has been chosen, sufficiently large so that no scale separation can be made between the two 

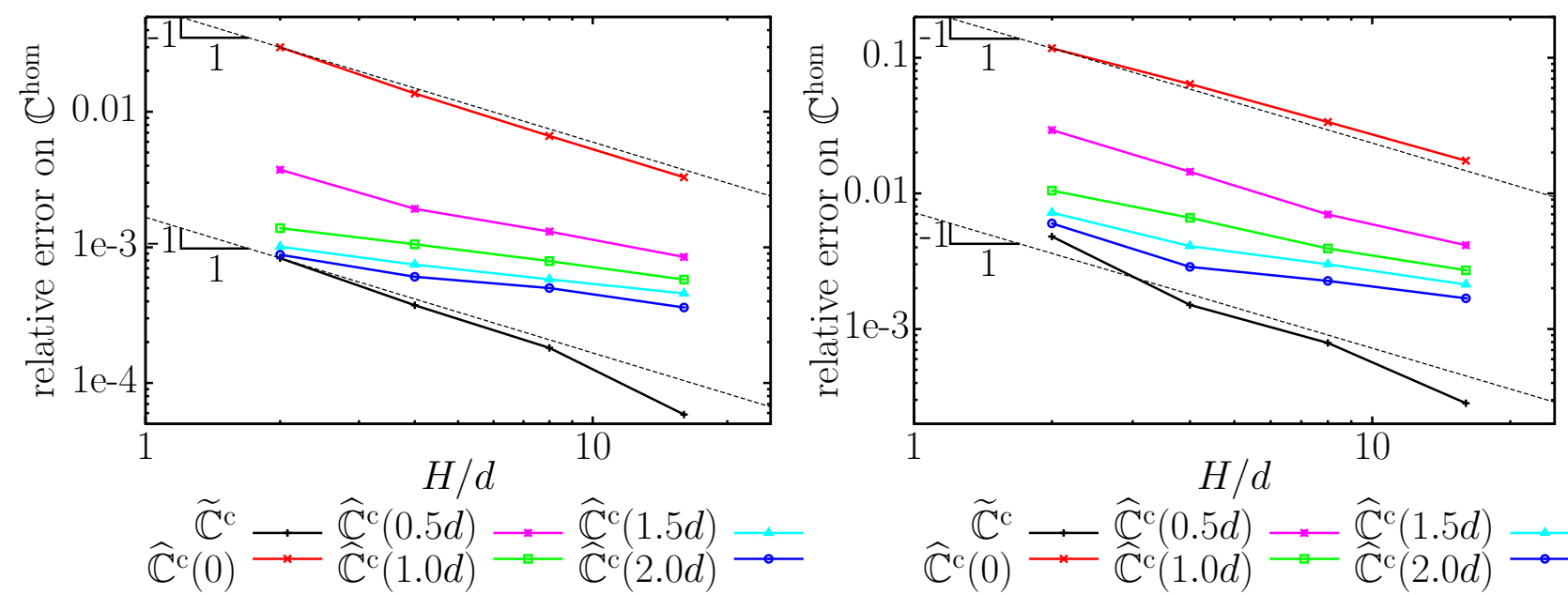

(a) contrast 1000

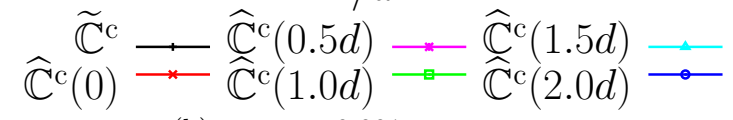

(b) contrast 0.001

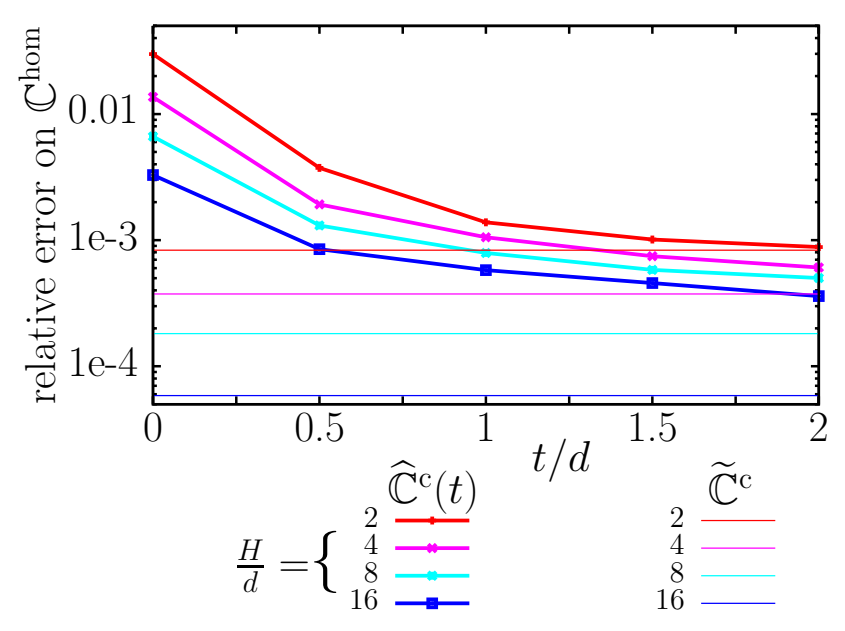

(c) contrast 1000

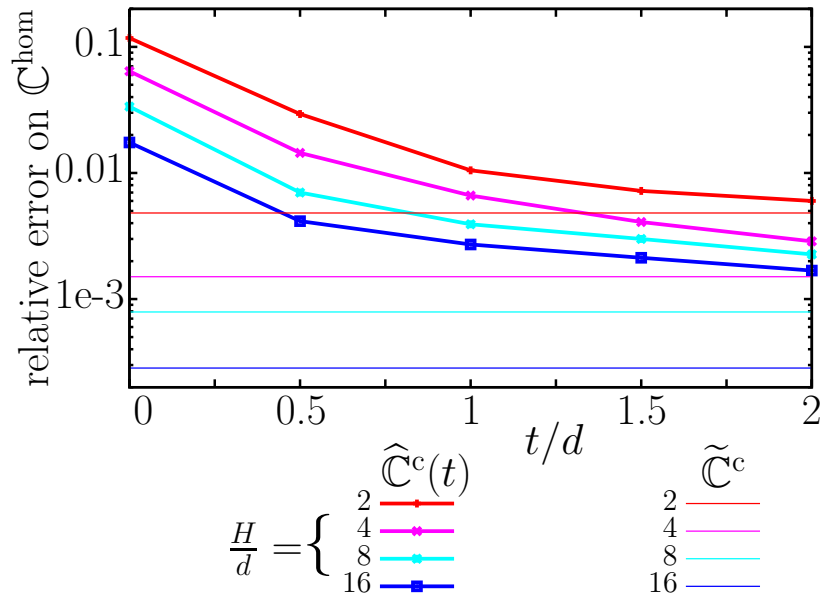

(d) contrast 0.001

Figure 10: Effect of oversampling size on the reduction of the error on $\mathbb{C}^{\text {hom }}$ due to non-conforming local problem boundary conditions, as a function of coarse discretization size to fiber diameter ratio $H / d$ (a), (b); as a function of oversampling thickness to fiber diameter ratio $t / d(\mathrm{c}),(\mathrm{d})$. 
types of heterogeneities and large enough to be computationally challenging for numerical methods. A RVE made of 49 large fibers and 3129 small fibers randomly positioned is then considered. Both fibers have a volume concentration of $15 \%$. All fibers have a shear modulus of 100 and a Poisson's ratio of 0.3 , while the matrix has a shear modulus of 1 and a Poisson's ratio of 0.3 . The fine discretization is set such that large fibers have a diameter $d_{\max }$ of 256 pixels and small fibers a diameter of $d_{\min } 32$ pixels. The whole fine discretization grid is made of $4096^{2}$ pixels. A direct computation of this discretized microstructure with a FFT-based method involves slightly more than 50 million degrees of freedom. The two step method is run with a coarse discretization size $H$ of twice the large fibers diameter and an oversampling thickness $t$ of half the large fibers diameter. The local problems each involve about 1.8 million degrees of freedom, resulting in a memory cost more than 28 times lower than the direct computation requires. Both direct and two step computations are carried out separately. The estimate $\widehat{\mathbb{C}}^{c}(t)$ of the consistent filtered stiffness is used for the coarse element properties. The two step method estimate of the homogenized stiffness results in a remarkably low relative error of $4.87 \times 10^{-3}$ on the homogenized stiffness obtained by the direct computation on the whole fine discretization grid.

While resorting to a FFT-based solver, the time improvements of the two step strategy appear only for very large discretization grids. However, memory cost, which is a major limiting factor, is much smaller since computations can be made separately.

\section{Conclusion}

The major contribution of this work was to establish a theoretical filtering framework for periodic homogenization. Starting from an initial homogenization problem, three types of filtered problems have been defined for any filter or unit mass function. First, a field of consistent filtered stiffness arose from the analysis, directly relating the filtered strain and stress solutions to the original problem. This stiffness, which conveys non-local information, is not symmetric. However, it is the only possible way to define macroscopically consistent problems, that is whose homogenization leads to exactly the same stiffness as the original homogenization problem one. Second, based on the strain energy filtering, a positive definite kinematic filtered stiffness is derived, whose homogenized stiffness is not macroscopically consistent but has a rigorous lower bound status. Third, the stress energy counterpart is proposed to derive a static filtered stiffness, yielding upper bounds on the homogenized stiffness. These two alternatives also allow to handle infinite contrasts. A remaining open question lies in the inversion of the filtered strain or stress localization tensors. Invertibility has not been proved but is necessary to the definitions.

Numerical simulations have next been carried out on simple composites made of cylindrical inclusions embedded in a homogeneous matrix. For these particular microstructures, the filtered stiffness fields have been observed to benefit from a smoothing effect. Namely, the filter size has an impact on the overall stiffness contrast while its anisotropy and regularity are transferred to the filtered stiffness field. Additionally, the property of consistency (12) and ordering (22) of the filtered problems have been assessed numerically. The non symmetric part of the consistent filtered stiffness field has been established to be necessary to obtain the right homogenized stiffness. In the mean time, the kinematic and static filtered stiffness fields provided sharp bounds.

Nonetheless, preliminary numerical studies of more complex microstructures suggest the filtered stiffness fields may be ill behaved, especially at high contrasts. Namely, sharp variations and extreme values of the discrete filtered stiffness arise in some regions. However, it has not been possible to establish whether these irregularities resulted from the initial problem discretization errors or not. It should be mentioned that for all tested microstructures and filters, the numerical inversion of the discrete filtered strain and stress localization tensors has always been possible.

In view of providing cost-efficient estimates of the filtered stiffness at sampling points positioned on a regular coarse grid, small local problems have been introduced. While the filtered stiffness does not suffer from non-conforming boundary conditions effect, effective stiffness estimates obtained from the resolution of a local problem are subjected to boundary conditions effect. An oversampling strategy has been devised to account for the non-locality of the filtered stiffness and reduce boundary effects. A thorough comparison has been proposed between the actual filtered stiffness and its estimates. Numerical results on composites 
made of inclusions embedded in a matrix showed the oversampling strategy allows to reduce these errors, gave detailed convergence and highlighted the effect of filter size.

Finally, a comparative study of the estimation of the homogenized stiffness from a two step method using the estimate or actual sampled filtered stiffness allowed to identify the leading term in the error between local problem boundary conditions issues and cell resonance effect.

As a concluding remark, the present numerical results, carried out on simple composites made of inclusions embedded in a matrix, should be put in perspective by a study of the effect of filtering on more complex microstructures, such as three dimensional bi-connex composites for which specific behaviors may appear.

\section{References}

[1] W. E, B. Engquist, X. Li, W. Ren, E. Vanden-Eijnden, Heterogeneous multiscale methods: A review, Communications in Computational Physics 2 (2007) 367-450.

[2] A. Abdulle, W. E, B. Engquist, E. Vanden-Eijnden, The heterogeneous multiscale method, Acta Numer. 21 (2012) 1-87.

[3] M. Geers, V. Kouznetsova, W. Brekelmans, Multi-scale computational homogenization: Trends and challenges, Journal of Computational and Applied Mathematics 234 (2010) 2175-2182.

[4] T. Hou, X.-H. Wu, A multiscale finite element method for elliptic problems in composite materials and porous media, Journal of Computational Physics 134 (1997) 169-189.

[5] A. Gloria, Numerical homogenization: Survey, new results, and perspectives, in: E. Cancès, E. S. Labbé (Eds.), ESAIM: Proceedings, volume 37, 2012, pp. 50-116.

[6] L. Durlofsky, Numerical calculation of equivalent grid block permeability tensors for heterogeneous porous media, Water Resources Research 57 (1991) 699-708.

[7] X. Wu, Y. Efendiev, T. Hou, Analysis of upscaling absolute permeability, Discrete and Continuous Dynamical SystemsSeries B 2 (2002) 185-204.

[8] X. Wen, L. Durlofsky, M. Edwards, Use of border regions for improved permeability upscaling, Mathematical Geology 35 (2003) 521-547.

[9] Y. Chen, L. Durlofsky, M. Gerritsen, X. Wen, A coupled local-global upscaling approach for simulating flow in highly heterogeneous formations, Advances in Water Resources 26 (2003) 1041-1060.

[10] X. Yue, W. E, The local microscale problem in the multiscale modeling of strongly heterogeneous media: Effects of boundary conditions and cell size, Journal of Computational Physics 222 (2007) 556-572.

[11] S. Baxter, L. Graham, Characterization of random composites using moving-window technique, Journal of Engineering Mechanics 126 (2000) 389-397.

[12] L. Graham-Brady, E. Siragy, S. Baxter, Analysis of heterogeneous composites based on moving-window techniques, Journal of Engineering Mechanics 129 (2003) 1054-1064.

[13] K. Acton, L. Graham-Brady, Elastoplastic mesoscale homogenization of composite materials, Journal of Engineering Mechanics 136 (2010) 613-624.

[14] W. Lian, Contribution à l'homogénéisation numérique du comportementélastique de matériaux à microstructure complexe caractérisés par imagerie, Ph.D. thesis, Ecole Centrale de Nantes, 2011.

[15] F. Di Paola, Modélisation multi-échelles du comportement thermo-élastique de composites à particules sphériques, Ph.D. thesis, Ecole Centrale Paris, 2010.

[16] X. Blanc, C. Le Bris, Improving on computation of homogenized coefficients in the periodic and quasi-periodic settings, Networks and Heterogeneous Media 5 (2010) 1-29.

[17] H. Moulinec, P. Suquet, A fast numerical method for computing the linear and non linear properties of composites, Comptes Rendus de l'Académie des Sciences 2 (1994) 1417-1423.

[18] S. Brisard, L. Dormieux, FFT-based methods for the mechanics of composites: A general variational framework, Computational Materials Science 49 (2010) 663-671.

[19] C. Huet, Application of variational concepts to size effects in elastic heterogeneous bodies, Journal of the Mechanics and Physics of Solids 38 (1990) 813-841.

[20] M. Ostoja-Starzewski, Micromechanics as a basis of random elastic continuum approximations, Probabilistic Engineering Mechanics 8 (1993) 107-114.

[21] W. E, P. Ming, P. Zhang, Analysis of the heterogeneous multiscale method for elliptic homogenization problems, J. Am. Math. Soc. 18 (2005) 121-156.

[22] A. Abdulle, Analysis of a heterogeneous multiscale FEM for problems in elasticity, Math. Models Methods Appl. Sci. 16 (2006) 615-635.

[23] P. Suquet, A simplified method for the prediction of homogenized elastic properties of composites with a periodic structure, Comptes-rendus de l'Académie des sciences série II 311 (1990) 769-774.

[24] Z. Hashin, S. Shtrikman, On some variational principles in anisotropic and nonhomogeneous elasticity, Journal of the Mechanics and Physics of Solids 10 (1962) 335-342.

[25] R. Zeller, P. Dederichs, Elastic constants of polycrystals, Physica Status Solidi B 55 (1973) 831-842.

[26] S. Brisard, L. Dormieux, Combining Galerkin approximation techniques and the principle of Hashin and Shtrikman 
to improve two FFT-based numerical methods for the homogenization of composites, Computer Methods in Applied Mechanics and Engineering 217-220 (2012) 197-212.

[27] R. Barrett, M. Berry, T. Chan, J. Demmel, J. Donato, J. Dongarra, V. Eijkhout, R. Pozo, C. Romine, H. Van der Vorst, Templates for the Solution of Linear Systems: Building Blocks for Iterative Methods, Society for Industrial and Applied Mathematics, 1994.

[28] C. Paige, M. Saunders, LSQR: An algorithm for sparse linear equations and sparse least squares, ACM Transactions on Mathematical Software 8 (1982) 43-71.

\section{Appendix A. Numerical resolution of a non symmetric linear elasticity homogenization prob- lem with FFT-based methods}

FFT-based homogenization methods, initially introduced by Moulinec and Suquet [17], are based on the introduction of a homogeneous reference material of uniform stiffness $\mathbb{C}^{0}$. The linear elastic behavior law can be artificially rewritten in a prestressed form

$$
\boldsymbol{\sigma}=\mathbb{C}: \epsilon=\mathbb{C}^{0}: \epsilon+\tau \text { with } \tau=\left(\mathbb{C}-\mathbb{C}^{0}\right): \epsilon,
$$

where the prestress $\boldsymbol{\tau}$ is referred to as a polarization field. The solution to the polarized problem with uniform stiffness can be obtained by means of the Green operator $\boldsymbol{\Gamma}_{0}[23]$ associated to the uniform stiffness $\mathbb{C}^{0}$

$$
\boldsymbol{\epsilon}=\boldsymbol{E}-\boldsymbol{\Gamma}_{0} * \boldsymbol{\tau}
$$

Then, the solution to the homogenization problem with imposed macroscopic strain $\boldsymbol{E}$ can be formally obtained by solving the Lippmann-Schwinger equation (A.3a), or equivalently but in terms of polarization, the strong form of the Hashin-Shtrikman variational principle (A.3b) [24, 25]:

$$
\begin{aligned}
& \boldsymbol{\epsilon}=\boldsymbol{E}-\boldsymbol{\Gamma}_{0} *\left[\left(\mathbb{C}-\mathbb{C}^{0}\right): \boldsymbol{\epsilon}\right], \\
& \left(\mathbb{C}-\mathbb{C}^{0}\right)^{-1}: \boldsymbol{\tau}+\boldsymbol{\Gamma}_{0} * \boldsymbol{\tau}=\boldsymbol{E} .
\end{aligned}
$$

The Lippmann-Schwinger equation (A.3a) remains valid even if $\mathbb{C}$ is not symmetric. The initial scheme proposed by Moulinec and Suquet [17] was to discretize (A.3b) by affecting a single value of the deformation field $\epsilon$ on each voxel of the regular grid. The discretized version of system (A.3b) is then solved using a fixed-point algorithm.

Recent advances $[18,26]$ shed a new light on FFT-based methods by bringing a clear distinction between the discretization and the resolution steps of equations (A.3). When $\mathbb{C}$ is definite positive, FFT-based methods may now benefit from a consistent Galerkin approximation framework which can lead to rigorous bounds on the homogenized modulii as well as consistent Green operators and stiffness field discretizations, which improve convergence. Even though the Galerkin approximation framework may not hold for non symmetric stiffness, we chose to use the discretization proposed by Brisard and Dormieux [26] which has a regularization effect.

Then, either the discretized Lippmann-Schwinger equation (A.3a) or its polarization formulation (A.3b) can be put in the form of a linear system of equations of finite dimension:

$$
\begin{aligned}
& \left(\mathbb{I}: \bullet+\boldsymbol{\Gamma}_{0} *\left[\left(\mathbb{C}-\mathbb{C}^{0}\right): \bullet\right]\right)(\boldsymbol{\epsilon})=\boldsymbol{E} \rightarrow[A]^{]^{\mathrm{s}}}[X]^{\epsilon}=[B], \\
& \left(\left(\mathbb{C}-\mathbb{C}^{0}\right)^{-1}: \bullet+\boldsymbol{\Gamma}_{0} * \bullet\right)(\boldsymbol{\tau})=\boldsymbol{E} \rightarrow[A]^{\mathrm{hs}}[X]^{\tau}=[B],
\end{aligned}
$$

where $[X]^{\epsilon}$ is the unknown vector made of all the components of every voxels of the discretized $\epsilon,[A]^{\text {ls }}$ is the matrix associated to the Lippmann-Schwinger operator in (A.4a) and the right hand side $[B]$ is made of the components of the macroscopic loading $\boldsymbol{E}$. Similarly, $[X]^{\tau}$ is the unknown vector made of all the components of every voxels of the discretized polarization field $\tau,[A]^{\text {hs }}$ is the matrix associated to the Hashin-Shtrikman 
operator in (A.4b). Note that if $\mathbb{C}$ is symmetric, $[A]^{\text {hs }}$ is symmetric by symmetry of the Green operator whereas $[A]^{] \mathrm{s}}$ is not.

Actually, the matrices $[A]$ are not explicitly written; only a method to compute the product $[A][X]$ given $[X]$ is necessary to the solver [18]. Any suitable iterative linear solver can be used to resolve the linear system (see Barrett et al. [27] for details). If the reference material is chosen stiffer or softer than any phase of the heterogeneous microstructure with symmetric positive definite stiffness $\mathbb{C}$, the linear system (A.4b) is symmetric positive definite and may be inverted with a Conjugated Gradient algorithm [18]. Otherwise, a saddle-point problem is to be solved, for example using the SymmLQ algorithm [27] if $\mathbb{C}$ is symmetric [26]. Whenever non symmetric stiffnesses such as the consistent filtered stiffness field are input, we propose to use the system (A.4a). Indeed, the system (A.4a) does not require the difference in stiffnesses inversion. The non positive definite and non symmetric linear system (A.4a) is solved with a non symmetric solver such as LSQR [28]. 